\title{
Graft-versus-host disease depletes plasmacytoid dendritic cell progenitors to impair tolerance induction
}

\author{
Yuanyuan Tian, ${ }^{1,2}$ Lijun Meng, ${ }^{2,3}$ Ying Wang, ${ }^{2,4}$ Bohan Li, ${ }^{3}$ Hongshuang Yu, ${ }^{2}$ Yan Zhou, ${ }^{5}$ Tien Bui, ${ }^{2}$ Ciril Abraham, ${ }^{2}$ Alicia Li, ${ }^{2}$ \\ Yongping Zhang, ${ }^{3}$ Jian Wang, ${ }^{3}$ Chenchen Zhao, ${ }^{6}$ Shin Mineishi, ${ }^{6}$ Stefania Gallucci, ${ }^{4}$ David Porter, ${ }^{7}$ Elizabeth Hexner, ${ }^{7}$ Hong Zheng, ${ }^{6}$ \\ Yanyun Zhang, ${ }^{1}$ Shaoyan $\mathrm{Hu},{ }^{3}$ and Yi Zhang ${ }^{2,4}$ \\ 'Shanghai Institute of Immunology, Institutes of Medical Sciences, Shanghai Jiao Tong University School of Medicine, Shanghai, China. ${ }^{2}$ Fels Institute for Cancer Research and Molecular Biology, Temple \\ University, Philadelphia, Pennsylvania, USA. ${ }^{3}$ Department of Hematology, Children Hospital, Soochow University, Suzhou, China. ${ }^{4}$ Department of Microbiology and Immunology, Temple University, \\ Philadelphia, Pennsylvania, USA. ${ }^{5}$ Biostatistics and Bioinformatics Facility, Fox Chase Cancer Center, Philadelphia, Pennsylvania, USA. ${ }^{6}$ Penn State Cancer Institute, Pennsylvania State University, Hershey, \\ Pennsylvania, USA. `Department of Medicine, Perelman School of Medicine, University of Pennsylvania, Philadelphia, Pennsylvania, USA.
}

\begin{abstract}
Graft-versus-host disease (GVHD) causes failed reconstitution of donor plasmacytoid dendritic cells (pDCs) that are critical for immune protection and tolerance. We used both murine and human systems to uncover the mechanisms whereby CVHD induces donor pDC defects. CVHD depleted Flt3-expressing donor multipotent progenitors (MPPs) that sustained pDCs, leading to impaired generation of pDCs. MPP loss was associated with decreased amounts of MPP-producing hematopoietic stem cells (HSCs) and oxidative stress-induced death of proliferating MPPs. Additionally, alloreactive T cells produced GMCSF to inhibit MPP expression of Tcf4, the transcription factor essential for pDC development, subverting MPP production of pDCs. GM-CSF did not affect the maturation of pDC precursors. Notably, enhanced recovery of donor pDCs upon adoptive transfer early after allogeneic HSC transplantation repressed CVHD and restored the de novo generation of donor pDCs in recipient mice. pDCs suppressed the proliferation and expansion of activated autologous T cells via a type I IFN signalingdependent mechanism. They also produced PD-L1 and LILRB4 to inhibit T cell production of IFN- $\gamma$. We thus demonstrate that GVHD impairs the reconstitution of tolerogenic donor pDCs by depleting DC progenitors rather than by preventing pDC maturation. MPPs are an important target to effectively bolster pDC reconstitution for controlling CVHD.
\end{abstract}

\section{Introduction}

Dendritic cells (DCs) play nonredundant roles in the regulation of both innate and adaptive immunity (1-3). However, inflammation, resulting from such conditions as chronic infection, autoimmunity, and tumor progression, may significantly affect DC generation and function (4-7). This consequence is observed clinically in graft-versus-host disease (GVHD) in individuals receiving allogeneic hematopoietic stem cell transplantation (allo-HSCT). Acute GVHD following allo-HSCT impairs the generation and function of donor DCs (8-14). GVHD is induced by alloreactive T cell responses that mediate tissue injury, primarily in the skin, liver, and gut (15). Clinical studies suggest that severe GVHD is tied to failed reconstitution of donor plasmacytoid DCs (pDCs) (8-10). Some preclinical studies suggest that GVHD may prevent the maturation of pDCs while promoting the generation of some suppressive precursor DCs (pre-DCs) (14). However, pDCs develop from hematopoietic stem cells (HSCs) through successive lineage commitment and differentiation steps: multipotent progenitors

Authorship note: YT and LM contributed equally to this work.

Conflict of interest: The authors have declared that no conflict of interest exists. Copyright: ( 2021, American Society for Clinical Investigation.

Submitted: January 27, 2020; Accepted: October 14, 2020; Published: January 4, 2021.

Reference information: J Clin Invest. 2021;131(1):e136774.

https://doi.org/10.1172/JCl136774.
(MPPs) $\rightarrow$ common DC progenitors (CDPs) $\rightarrow$ pre-DCs (3). Many studies indicate that the aforementioned inflammatory responses may inhibit HSCs' and DC progenitors' regenerative capacity (16-19). Whether GVHD impairs the generation of DC progenitors to induce pDC defects remains unknown.

Addressing this question is important to improving the efficacy of allo-HSCT. pDCs are critical for regulating both immune protection and tolerance. pDCs specialize in rapidly producing large amounts of IFN- $\alpha$, protecting against pathogenic infection (20). In spite of their importance in mediating autoimmunity, pDCs are also known to be tolerogenic (21-25). pDCs can suppress $\mathrm{T}$ cell responses and repress alloimmunity $(4,14,26-28)$. In mice receiving allo-HSCT, the transfer of host-type pDCs expressing CCR9 (CCR9 ${ }^{+}$) suppressed GVHD (26). However, since these $\mathrm{CCR}^{+} \mathrm{pDCs}^{\mathrm{Pae}}$ derived from mice bearing Flt3 ligand-producing (Flt3L-producing) melanoma (26), the pDCs could, problematically, be programmed to be tolerogenic during tumor progression. In fact, host mature pDCs are found to elicit GVHD in mice (29). Notably, both clinical and preclinical studies suggest that donor pDCs from bone marrow (BM) grafts can reduce the severity of GVHD $(14,27,30)$. However, the precise impact normal donor-derived pDCs have on GVHD has yet to be determined. We reason that if donor pDCs can suppress GVHD, early recovery of donor pDCs after allo-HSCT may break the feed-forward GVHD cascade that characterizes the disease's progression. 
A
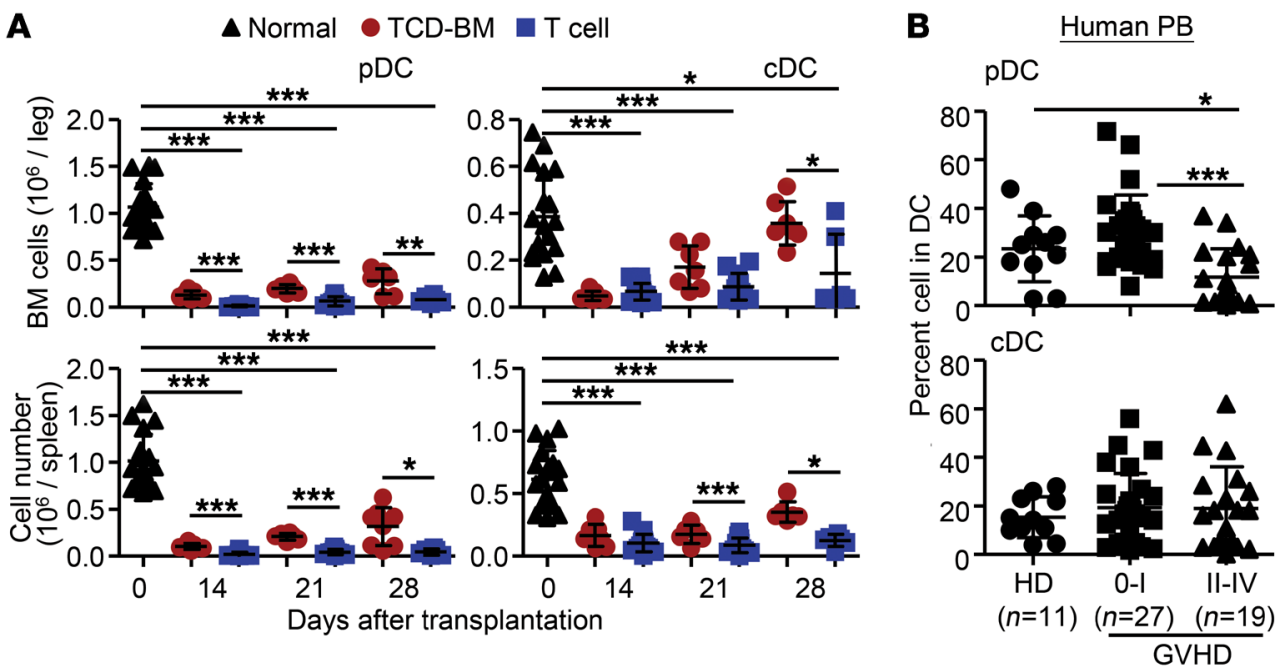

D
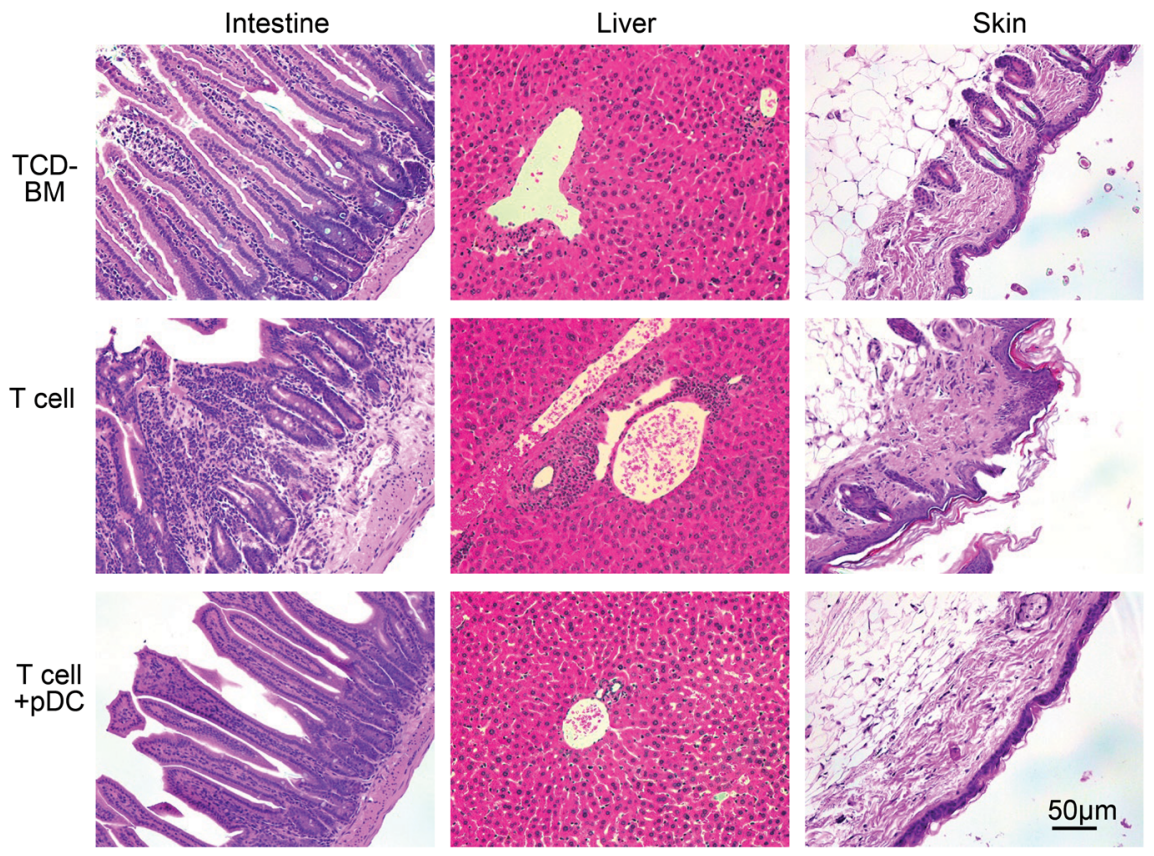

C $\quad$ TCD-BM $(n=8)$

-T cell $(n=12)$

+ T cells + pDC $(n=20)$

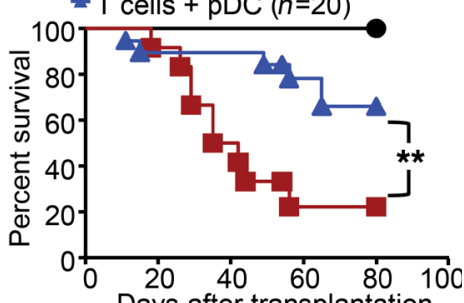

Days after transplantation
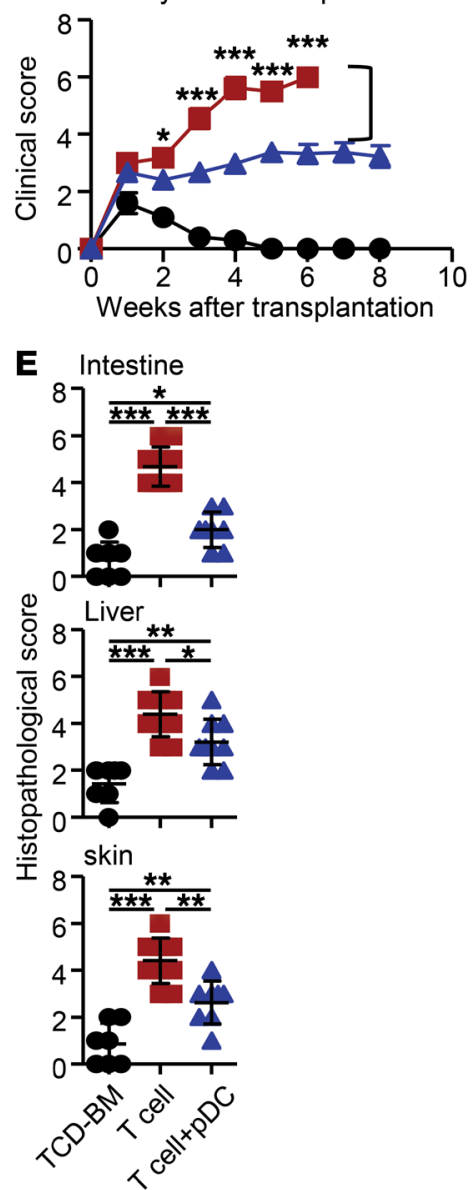

Figure 1. Donor pDCs inhibit a feed-forward cascade reaction of GVHD. (A) C57BL/6 (B6) mouse-derived T cell-depleted bone marrow (TCD-BM) (5 $\times 10^{6}$ cells) was transplanted, with or without $C D 4^{+} T$ cells $\left(5 \times 10^{5}\right)$, into lethally irradiated BALB/c mice to induce GVHD. Graphs show pDC and cDC numbers in the BM and spleen. Each group contained 4 to 10 mice. (B) Graphs show pDCs and cDCs in PB obtained from healthy donors (HD, $n=11)$ and patients undergoing allo-HSCT $(n=46)$. Samples were collected at the time of GVHD onset, between 21 and 70 days after transplantation. Patients with grade IIIV CVHD ( $n=19)$, patients with or without grade I GVHD $(n=27)$. (C) Donor-type pDCs were generated from B6 BM, activated overnight by TLR4 (LPS) and TLR7/8 (R8484) agonists, thoroughly washed using PBS, and transferred to lethally irradiated BALB/C mice receiving B6 T cells plus TCD-BM in 3 doses on days 0,1 , and $2\left(1 \times 10^{6} \mathrm{pDCs} /\right.$ day). Survival and clinical score were monitored over time. (D and E) Tissues were collected on day 22 after transplantation, or later (upon recipient mouse termination). (D) Images (H\&E stained) were obtained at $\times 200$ magnification. (E) Graphs show the histological score of inflammation in the intestine, liver, and skin. Data are presented as mean \pm SD. Multiple comparisons were evaluated by 1-way ANOVA with Bonferroni's multiple-comparison test (A and $\mathbf{E}$ ) and Kruskal-Wallis with Dunn's multiple-comparison test (B). Survival comparisons were evaluated by log-rank test (C, upper panel) and clinical score (mean $\pm \mathrm{SD}$ ) comparisons by 2-way ANOVA and unpaired $t$ test (D, lower panel). Data are representative of 2 to 3 independent experiments (E). ${ }^{*} P<0.05$; ${ }^{* *} P<0.01$; ${ }^{* *} P<0.001$.

Herein, we demonstrate for the first time to our knowledge that alloreactive $\mathrm{T}$ cell responses and GVHD mediate donor pDC defects by depleting donor MPPs and subverting MPP production of pDCs. MPP depletion is the culmination of multiple discrete outcomes, including decreased numbers of donor HSCs that give rise to MPPs, increased MPP death, and skewed MPP differentiation. Furthermore, enhanced recovery of pDCs early after allo-HSCT reduces GVHD and improves de novo generation of donor pDCs. Our findings open up new routes to investigate the molecular mechanisms by which GVHD causes dysregulated reconstitution of donor DCs. 


\section{Results}

GVHD impairs the reconstitution of donor pDCs that can potently suppress GVHD. We characterized the effect of GVHD on donor DC reconstitution both in mice and humans. DCs can be broadly categorized into 2 groups: conventional DCs (cDCs) and pDCs $(5,31)$. Murine pDCs and cDCs were defined by their surface phenotype: $\mathrm{CD} 11 \mathrm{c}^{+} \mathrm{B} 22 \mathrm{O}^{+}$Siglec- $\mathrm{H}^{+}$and $\mathrm{CD} 11 \mathrm{c}^{+} \mathrm{B} 22 \mathrm{O}^{-} \mathrm{Si}-$ glec- $\mathrm{H}^{-}$, respectively (Supplemental Figure 1, A and B; supplemental material available online with this article; https://doi. org/10.1172/JCI136774DS1). In mice, donor T cells caused lethal GVHD (Supplemental Figure 1C), which was associated with dramatically decreased frequency and numbers of pDCs and cDCs in the BM and spleen throughout the first 28 days after transplantation, when the majority of $\mathrm{T}$ cell recipients died from GVHD (Figure 1A). However, compared with normal mice, mice receiving $\mathrm{T}$ cell-depleted $\mathrm{BM}$ (TCD-BM) only achieved significant $\mathrm{CDC}$ recovery in the BM 28 days after transplant; notably, pDC recovery continued to be suppressed (Figure 1A). These data suggest that while defects in donor DC reconstitution may be largely attributed to GVHD, the procedure of TCD-BM may have a negative impact on donor $\mathrm{pDC}$ reconstitution.

To determine whether GVHD reduced circulating pDCs in humans, we obtained peripheral blood (PB) from pediatric patients (Supplemental Table 1) and adult patients (Supplemental Table 2) at the onset of GVHD. Human PB pDCs are Lin ${ }^{-} \mathrm{HLA}^{-\mathrm{DR}^{+}}$ $\mathrm{CD} 1 \mathrm{c}^{-} \mathrm{CD} 123^{\text {hi }}$ cells, whereas $\mathrm{CD} 1 \mathrm{c}^{+} \mathrm{cDCs}$ are characterized as

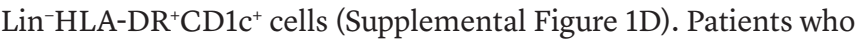
developed grade II-IV acute GVHD $(n=19)$ had approximately two-thirds fewer pDCs than patients without GVHD or with mild GVHD $(n=27)$ (Figure 1B). No significant correlation between GVHD and CD1c $\mathrm{c}^{+} \mathrm{cDCs}$ was observed across these 2 groups (Figure 1B). Notably, for both pediatric and adult cohorts, allo-HSCT patients with grade II-IV GVHD had fewer blood pDCs than patients with grade 0-I GVHD (Supplemental Figure 1, E and F). Thus, pDCs are more sensitive than cDCs to GVHD-induced suppression in patients undergoing allo-HSCT.

To assess the role of donor-type pDCs in the modulation of GVHD, we generated pDCs from the BM of B6 mice, as described previously (32). After activation with LPS plus R848, we sorted MHC- $\mathrm{II}^{+} \mathrm{CD} 11 \mathrm{c}^{+} \mathrm{CD} 11 \mathrm{~b}^{-} \mathrm{B} 22 \mathrm{O}^{+}$pDCs (Supplemental Figure $1 \mathrm{G}$ ) and confirmed their ability to produce high levels of Ifna and Ifnb upon $\mathrm{CpG}$ stimulation (Supplemental Figure $1 \mathrm{H}$ ). Infusion of these donor-type pDCs on days 0,1 , and 2 after transplant reduced GVHD in BALB/c recipients, as evidenced by the significantly decreased clinical scores and improved overall survival of the allo-HSCT mice (Figure 1C). Histological examination confirmed decreases in liver, skin, and intestine inflammation in pDC-treated recipients compared with untreated, control $\mathrm{T}$ cell recipients (Figure 1, D and E). The low frequency of DCs in normal BM or G-CSF-mobilized blood has hampered attempts to harness pDCs for GVHD prevention. Upon titrating the numbers of donor $\mathrm{pDCs}$, we observed that infusion of as few as $0.2 \times 10^{6}$ donor DCs was sufficient to induce potent GVHD suppression in mice (Supplemental Figure 2A). We confirmed that donor pDC treatment suppressed GVHD in BDF1 mice receiving MHC- and miHA-mismatched B6 $\mathrm{CD}^{+} \mathrm{T}$ cells plus $\mathrm{CD} 8^{+} \mathrm{T}$ cells, with significantly improved survival rates (Supplemental Figure 2B), ruling out a model-spe- cific effect of donor pDC therapy. These data suggest that GVHD impairs donor pDC reconstitution and that the recovery of donor pDCs early after allo-HSCT may inhibit the disease.

GVHD reduces the quantity and quality of donor-derived $D C$ progenitors. We next examined the impact of GVHD on the generation of DC progenitors (e.g., MPPs and CDPs) both in murine and human systems. We defined murine MPPs by their surface phenotype Lin $^{-}$Sca $1^{\text {hi }}$ - - kit $^{\text {hi }}$ CD150-Flt3 ${ }^{+}$(CD150-Flt3 ${ }^{+}$LSK cells), and CDPs as Lin $^{-} \mathrm{Sca1}^{-} \mathrm{c}-\mathrm{kit}^{\mathrm{int}} \mathrm{Flt}^{+}{ }^{+}$(Supplemental Figure 3A). As compared with TCD-BM mice and normal donor mice, GVHD mice produced 2- to 3-fold fewer MPPs and CDPs after allo-HSCT (Figure 2A and Supplemental Figure 3A). To test the capacity of GVHD MPPs and CDPs to produce pDCs, we purified them from GVHD mouse BM and stimulated them with Flt3L plus SCF. Both GVHD MPPs and CDPs showed a decreased capacity to produce pDCs compared with normal MPPs and CDPs (Figure 2B and Supplemental Figure 3B). Thus, GVHD-mediated pDC defects are likely to be associated with the impairment of DC progenitor quantity and quality. Human MPPs were defined as CD34 $4^{+} \mathrm{CD} 38^{-}$ $\mathrm{CD}^{-} \mathrm{CD}^{-} 5 \mathrm{RA}^{-}$and $\mathrm{CDPs}$ as $\mathrm{CD}^{-} 4^{+} \mathrm{CD} 38^{+} \mathrm{CD} 10^{-} \mathrm{CD} 45 \mathrm{RA}^{+}$ $\mathrm{CD}_{123}{ }^{+} \mathrm{CD} 115^{-}$(Figure 2C) (33). We observed significantly fewer MPPs and CDPs in patients with grade II-IV GVHD compared with patients without GVHD or with mild GVHD (Figure 2, C and D, and Supplemental Table 3). Further studies are needed to test whether GVHD patients produce MPPs and CDPs that have altered pDC production abilities.

MPPs sustain pDCs but are depleted during GVHD. To ascertain at which pDC development stage(s) GVHD impairs pDC reconstitution, we sorted HSCs, MPPs, and CDPs from the BM of normal mice (Figure 3A). Ex vivo stimulation with Flt3L showed that MPPs produced approximately 60-fold more pDCs than CDPs did throughout day 9 of culture (Figure 3B). CDPs rapidly produced $\mathrm{pDCs}$ at earlier rather than later time points (Figure 3B). This suggests that MPPs play a larger role in sustaining pDCs. There were significant increases in HSCs' production of pDCs at later time points in cultures (Figure 3B), but with a 2- to 3-fold lower frequency compared with MPPs' production (Figure $3 \mathrm{~B}$ ), suggesting the need for a longer time frame for HSC differentiation into pDCs.

We therefore focused on investigating the effect of GVHD on MPPs. Flt3 expression appears first in MPPs (34). Based on Flt3 expression levels, we separated MPP into 2 subsets: Flt ${ }^{\text {hi }}$ and moderately Flt3-positive (Flt3 ${ }^{\text {mod}}$ ) MPPs (Figure 3C). Compared with Flt $3^{\text {mod }}$ MPPs, Flt $3^{\text {hi }}$ MPPs produced approximately 3-fold more pDCs (Figure 3D), had a greater capacity to proliferate in culture (Figure 3E), and expressed lower levels of $p 16^{\text {Ink4a }}$, Spi1, and Irf8 (Figure 3F). p16 ${ }^{\text {Ink4a }}$ encodes a cyclin-dependent kinase inhibitor that limits the hematopoietic stem/progenitor cell (HSPC) replicative life span (35), whereas Spi1 (encoding Pu.1) and Irf8 are expressed in cells that are further differentiated $(36,37)$. This suggests that Flt $3^{\text {hi }}$ MPPs are less differentiation-prone than Flt ${ }^{\text {mod }}$ MPPs. GVHD depleted Flt $3^{\text {hi }}$ MPPs (Figure 3, G and H) and reduced the number of Flt $3^{\text {mod }}$ MPPs in the BM (Figure $3 \mathrm{H}$ ). Furthermore, at a cellular level, GVHD Flt3 ${ }^{\text {mod }}$ MPPs produced 3- to 4-fold fewer pDCs than did normal MPPs (Figure 3I). Collectively, these results show that GVHD impairs the generation and maintenance of both MPP subsets, with the largest effect on Flt $3{ }^{\text {hi }}$ MPPs. 
A

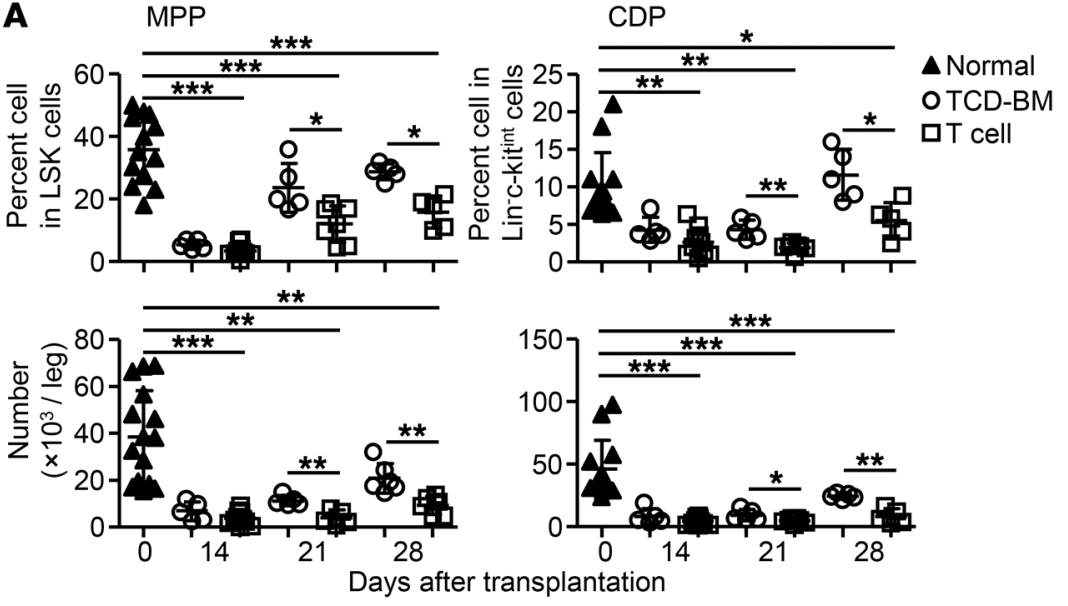

B
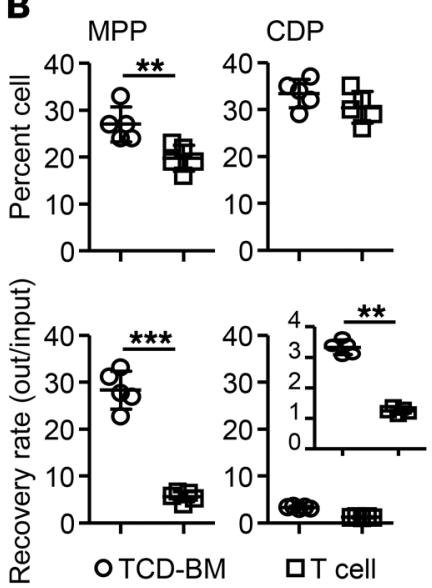

C
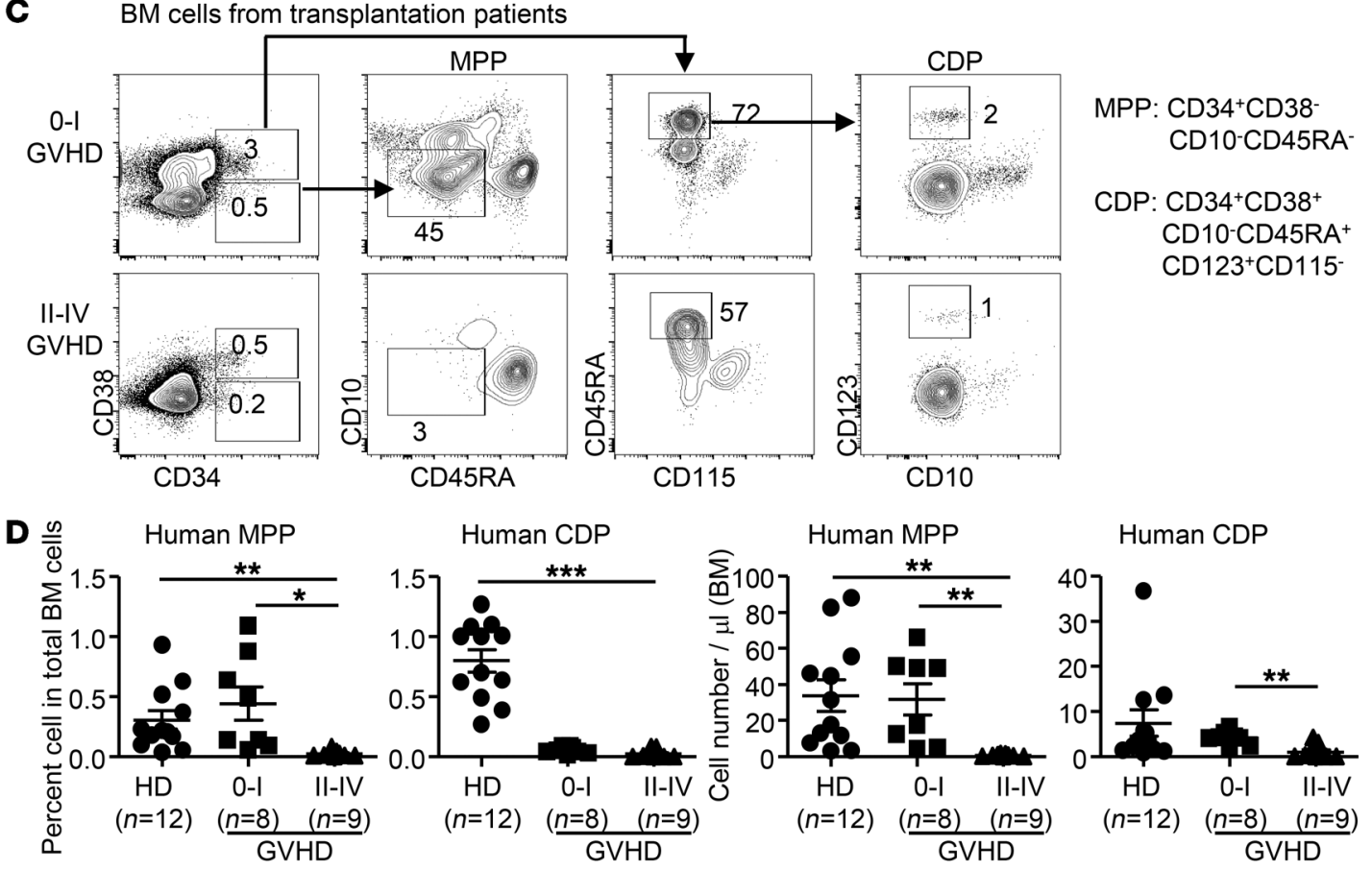

Figure 2. GVHD causes loss of DC progenitors. (A and B) B6 TCD-BM $\left(5 \times 10^{6}\right.$ cells) was transplanted with or without CD4+ $\mathrm{T}$ cells $\left(5 \times 10^{5}\right)$ into lethally irradiated BALB/c mice to induce GVHD. (A) Percentages and number of MPPs and CDPs in the BM from normal, TCD-BM, and T cell recipients; each group contained 4 to 10 mice pooled from at least 3 independent experiments. (B) MPPs and CDPs were isolated by FACS from normal and GVHD mice (CD45.2+) and cultured with feeder cells (BM from B6/SJL mice, CD45.1 $1^{+}$in the presence of Flt3L plus SCF. MPPs and CDPs were cultured for 9 days and 3 days, respectively. Percentages and numbers of pDCs were measured. (C and $\mathbf{D})$ BM cells were obtained from HD and allo-HSCT patients. (C) Plots show pDC and CDC gating strategy. Plots show phenotypes of human CD34+ HSPCs from HD $(n=12)$, grade 0-I GVHD patients $(n=7)$, and grade II-IV GVHD patients $(n=$ 8). (D) Graphs show the percentages of human MPPs and CDPs from healthy donors (HD) ( $n=12)$, grade 0-I GVHD patients ( $n=8)$, and grade II-IV GVHD patients $(n=9)$. Multiple comparisons were evaluated by 1-way ANOVA with Bonferroni's multiple-comparison test (A), 2-group comparisons by unpaired $t$ test (2-tailed) (B), and multiple comparisons by Kruskal-Wallis test with Dunn's multiple-comparison test (D). Data shown are mean \pm SD. Results shown in $\mathbf{A}$ and $\mathbf{B}$ are representative of 3 independent experiments. ${ }^{*} P<0.05 ;{ }^{* *} P<0.01 ;{ }^{* *} P<0.001$.

GVHD reduces the number of self-renewing HSCs. Loss of Flt $3^{\text {hi }}$ MPPs during GVHD may be the result of their decreased generation, impaired maintenance, and/or skewed differentiation. HSCs are known to have a population enriched in Flt3- LSK cells that can give rise to Flt3 ${ }^{+}$MPPs $(38,39)$. Compared with normal murine Flt3- ${ }^{-}$LSK cells, GVHD Flt3 ${ }^{-}$LSK cells downregulated $M p l$, Fbw7, Nr4a1, Tie1, and Klf6 (Figure 4A), genes critical for maintaining a quiescent and self-renewing HSC phenotype $(40,41)$. Gene set enrichment analysis showed that GVHD Flt3- LSK cells decreased the expression of genes associated with long-term HSCs (LT-HSCs) but increased those associated with short-term HSCs (ST-HSCs) (Figure 4B). Similar to these observations in mice, human patients with grade II-IV GVHD had 3-fold fewer CD34+ HSPCs than patients with grade O-I GVHD (Supplemental Figure 4). Lower numbers of $\mathrm{CD}^{2} 4^{+}$cells in the BM of patients with severe GVHD was not the result of infusing fewer CD34+ cells (Supplemental Table 3). These patients did not receive posttransplant cyclophosphamide or anti-thymocyte globulin treatment. Thus, GVHD also causes HSPC decreases in patients. 
A
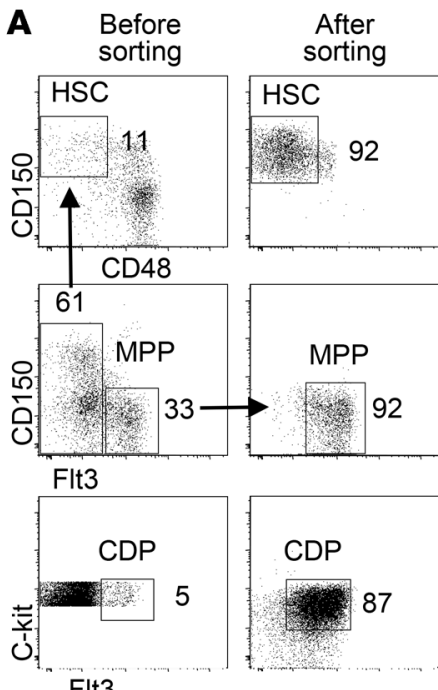

FIt3

Spi1
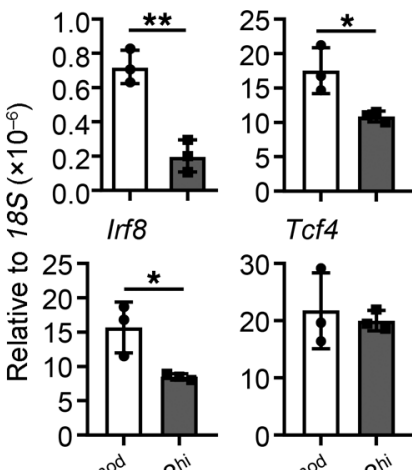

$<+3^{m n^{d}}<+3^{n i}$

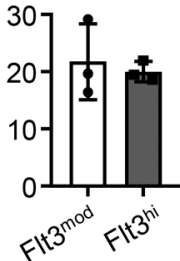

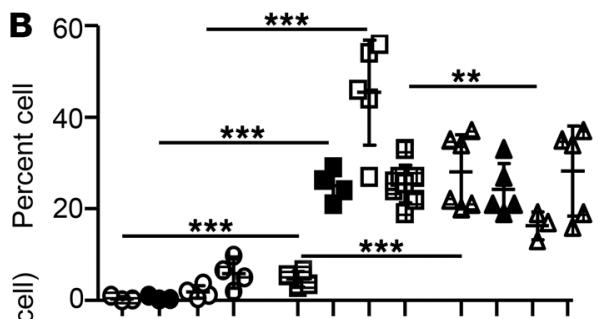
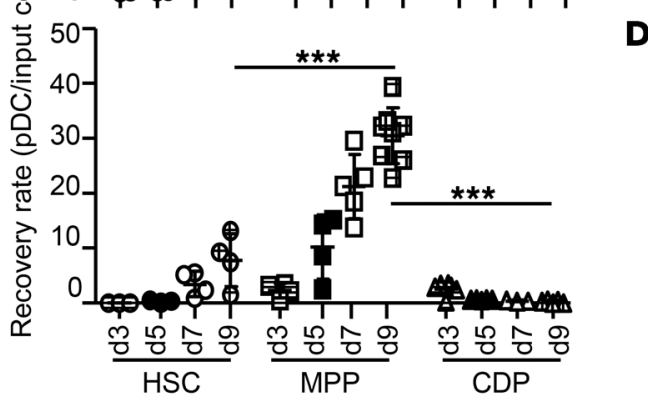

G

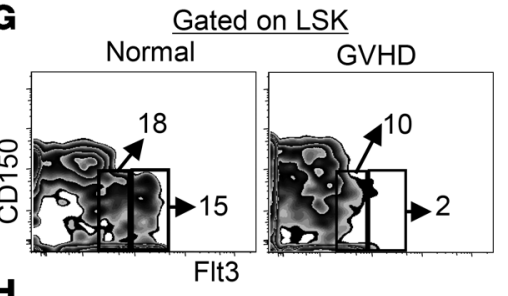

H
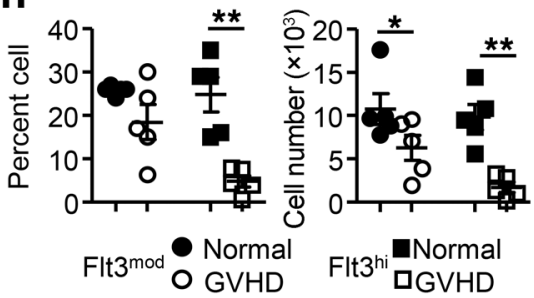

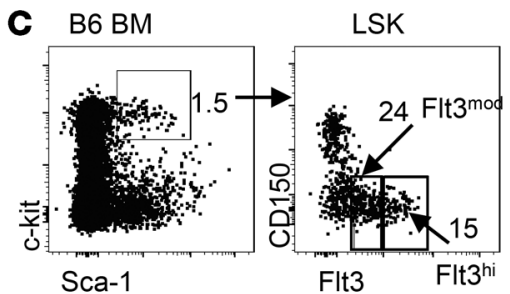

D

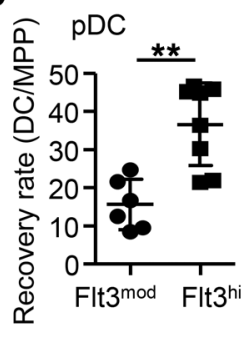

$\mathbf{E}$

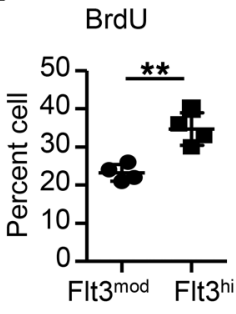

Figure 3. GVHD impairs both the quantity and quality of MPPs that sustain pDCs. HSCs (CD150+CD48- LSK cells), MPPs, and CDPs were isolated by FACS from the BM of normal mice (A) and cultured in the presence of Flt3L plus SCF to induce pDC generation (B). (C) Plots show Flt3 expression on the surface of MPPs. (D) Generation of pDCs from Flt3 ${ }^{\text {hi }}$ MPPs and Flt3 $3^{\bmod }$ MPPs in cultures stimulated with Flt3L plus SCF. (E) Proliferation capacity of Flt3 ${ }^{\text {hi }}$ MPPs and Flt3 ${ }^{\text {mod }}$ MPPs in vivo indicated by BrdU. (F) Real-time RT-PCR assay revealed the relative expression of indicated genes in freshly isolated Flt $3^{\text {hi }}$ MPPs and Flt3 ${ }^{\bmod }$ MPPs (mean \pm SD). (G-I) B6 TCD-BM $\left(5 \times 10^{6}\right.$ cells) was transplanted with or without CD4 ${ }^{+} \mathrm{T}$ cells $\left(5 \times 10^{5}\right)$ into lethally irradiated BALB/c mice to induce GVHD. BM cells were recovered from these mice 21 days after transplantation. Plots show the fraction of Flt $3^{\text {hi }}$ MPPs and Flt $3^{\text {mod }}$ MPPs in the BM of normal and GVHD mice ( $n=5$ per group) (C) and graphs show the frequency and numbers of BM of Flt $3^{\text {hi }}$ MPPs and Flt ${ }^{\text {mod }}$ MPPs (H). (I) Flt $3^{\text {mod }}$ MPPs were sorted from the BM of normal and CVHD mice and cultured in the presence of Flt3 plus SCF for 9 days. Graphs show the percentage and number of pDCs and $\mathrm{CDC}$ s derived from the culture. Data shown are mean \pm SD. Results are representative of at least 2 independent experiments, with triplicates in each group. Multiple comparisons were evaluated by 1-way ANOVA together with Bonferroni's multiple-comparison test (B and $\mathbf{H})$, and 2-group comparisons by 2-tailed unpaired $t$ tests $(\mathbf{D}-\mathbf{F}$ and $\mathbf{I}) .{ }^{*} P<0.05 ;{ }^{* *} P<0.01 ;{ }^{* *} P<0.001$.

GVHD Flt3- LSK cells are a heterogeneous population. The decrease in expression of genes associated with LT-HSCs might reflect a decrease in the number of LT-HSCs. GVHD mice had approximately 3-fold fewer $\mathrm{CD} 150^{+} \mathrm{CD} 48^{-}$LT-HSCs compared with naive mice and TCD-BM recipient mice (Figure 4, C and D). Notably, we observed no significant differences between normal HSCs' (CD45.1) and GVHD HSCs' (CD45.2) in vivo production of MPPs on days 15 and 34 after adoptive transfer (Figure 4, $\mathrm{E}$ and $\mathrm{F}$ ). Thus, GVHD is shown to cause a dramatic reduction in HSC numbers, which is consistent with reports that inflammation induces the loss of HSCs $(17-19,42)$. However, at a cellular level, GVHD HSCs retain their ability to produce MPPs. Reduced numbers of HSCs during GVHD may contribute to decreases in MPPs.
Alloreactive T cells induce MPP loss and polarize MPP differentiation. We next examined the impact of GVHD on MPP maintenance. Inflammation is shown to induce hyperactivation of $\mathrm{mTOR}$ in HSPCs, leading to HSPC loss (19). Many factors influence the activation of the mTOR pathway, which can be measured by increases in phosphorylated S6K1 (p-S6K1) $(19,43)$. Compared with normal MPPs, GVHD MPPs had significantly higher levels of p-S6K1 and reactive oxygen species (ROS) (Figure 5, A and B), suggesting increased mTOR activity in GVHD MPPs. In vivo administration of the mTOR inhibitor rapamycin led to a 5 -fold greater MPP frequency in GVHD mice (Figure 5C). However, the treatment did not improve donor $\mathrm{pDC}$ recovery in these mice (data not shown), likely due to rapamycin inhibition of pDC devel- 
A

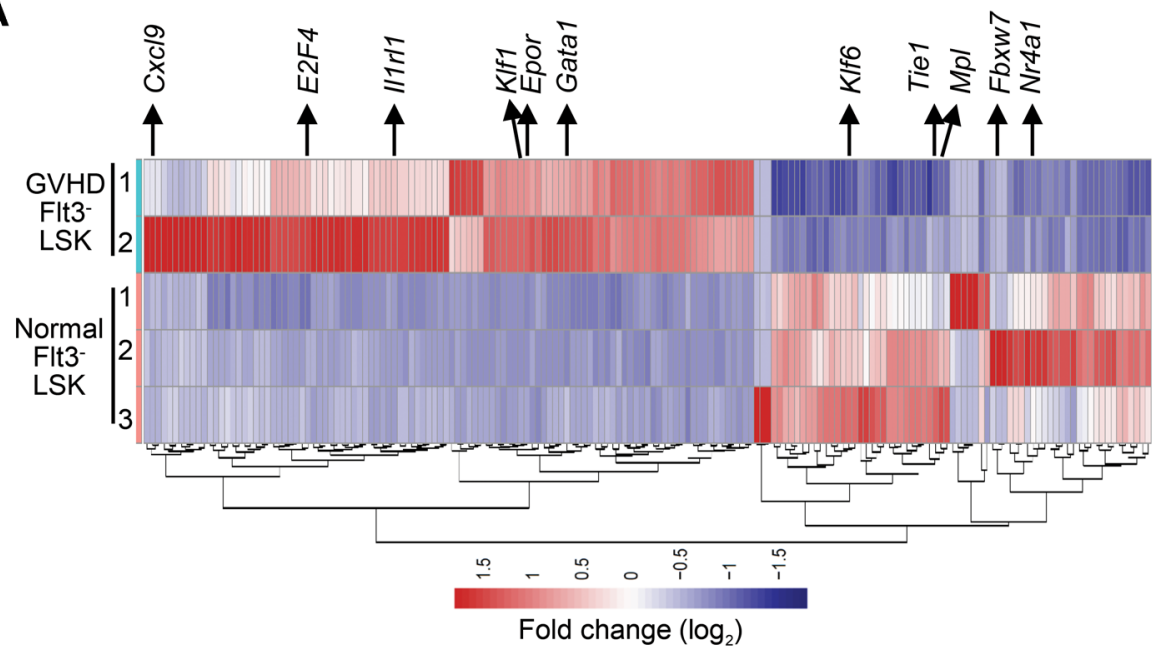

B

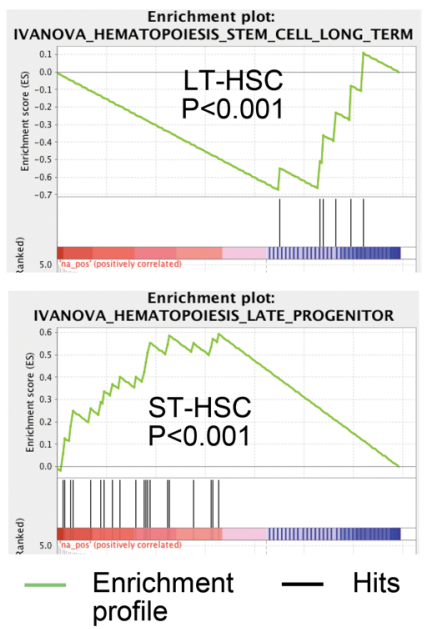

C

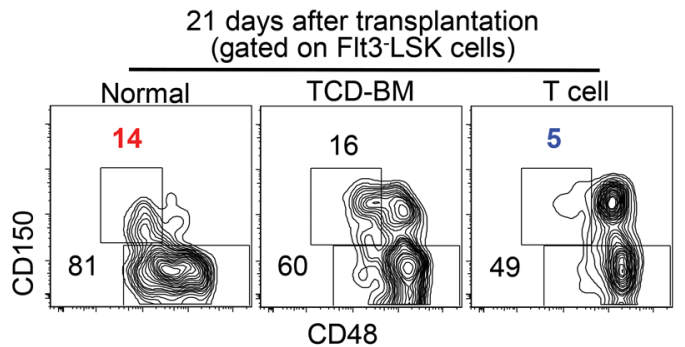

E

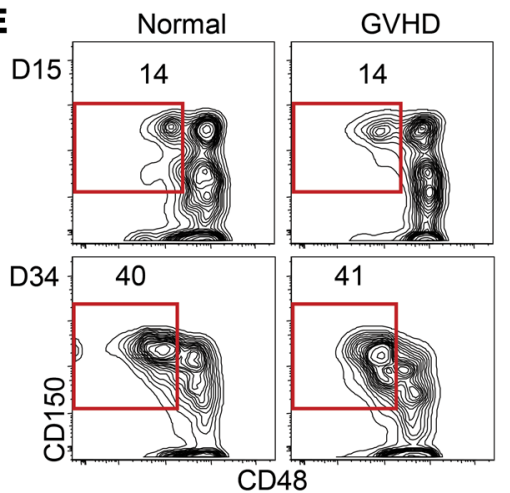

D

LT-HSC

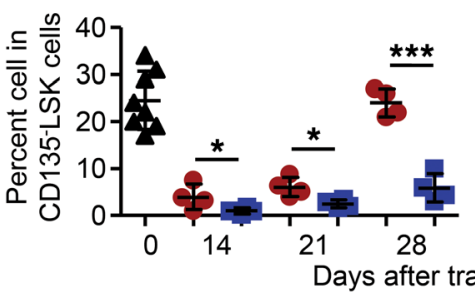

LT-HSC

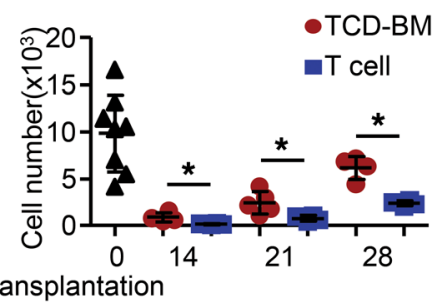

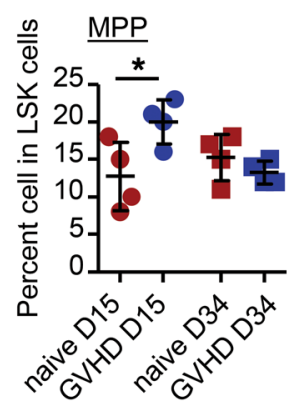

Flt3

Figure 4. LT-HSCs are decreased in CVHD but retain the capacity to produce MPPs. B6 TCD-BM, together with or without T cells, was transferred into lethally irradiated BALB/C mice. (A and B) Flt3- LSK cells were highly purified from the BM of normal and GVHD mice 21 days after transplantation. RNA was extracted from these HSPCs for RNA-seq analysis. (A) Heatmap shows genes differentially expressed in normal and GVHD FIt3- LSK cells. (B) Cene set enrichment analysis of the characteristics of genes associated with HSCs. (C-F) BM cells from normal, TCD-BM recipients, and T cell recipients were stained with a panel of antibodies that recognize stem cells. (C) Percentage and numbers of LT-HSCs in the BM were examined. (D) LT-HSCs (CD45.2+) were purified from the BM of normal and GVHD mice 21 days after transplantation and adoptively transferred into sublethally irradiated B6/SJL mice. (E and F) After an additional 15 and 34 days, cells from the BM of these secondary recipients were examined to monitor the regeneration of HSCs and Flt3 ${ }^{+}$MPPs. Multiple comparisons were evaluated by 1-way ANOVA with Bonferroni's multiple-comparison test (D-F). Data shown are mean \pm SD. Results shown in C-F are representative of at least 2 independent experiments. ${ }^{*} P<0.05$; ${ }^{* *} P<0.001$.

opment (44). To combat this, we cultured GVHD MPPs with Flt3L plus SCF without rapamycin to test their survival capability during pDC formation. Compared with normal MPPs, there were approximately $30 \%$ and $50 \%$ reductions in GVHD MPP recovery rate on days 3 and 9, respectively, compared with normal MPPs (Figure 5, D and E). The decreased recovery rate of cultured GVHD MPPs was associated with significantly increased cell death in dividing MPPs (Figure 5D) and MPP-derived pDCs (Figure 5E). Addition of the antioxidant $N$-acetyl cysteine (NAC), which antagonizes ROS induced by hyperactive mTOR effects, to GVHD MPP cultures markedly reduced the frequency of dead pDCs (Figure 5F). Thus, activated mTOR in MPPs may play a critical role in mediating the loss of pDC-forming MPPs during GVHD.

Both IFN- $\gamma$ and TNF- $\alpha$ can inhibit HSC proliferation (17, 18 ), and GM-CSF is important for inducing the generation of monocyte DCs (mo-DCs) (4). Alloreactive T cells pro- 
A

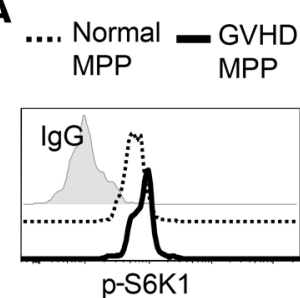

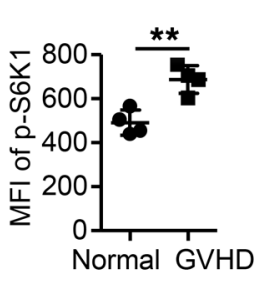

B

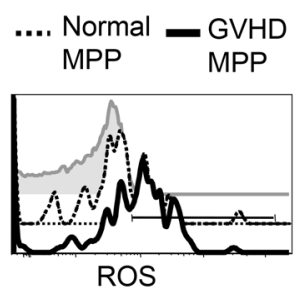

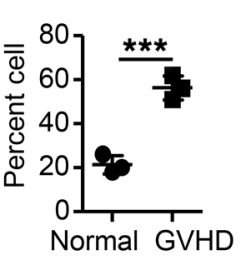

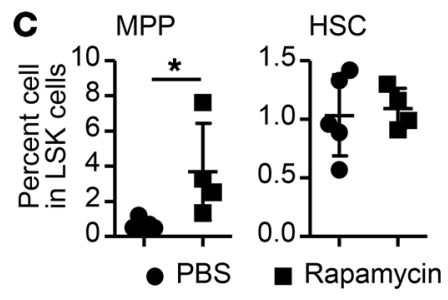

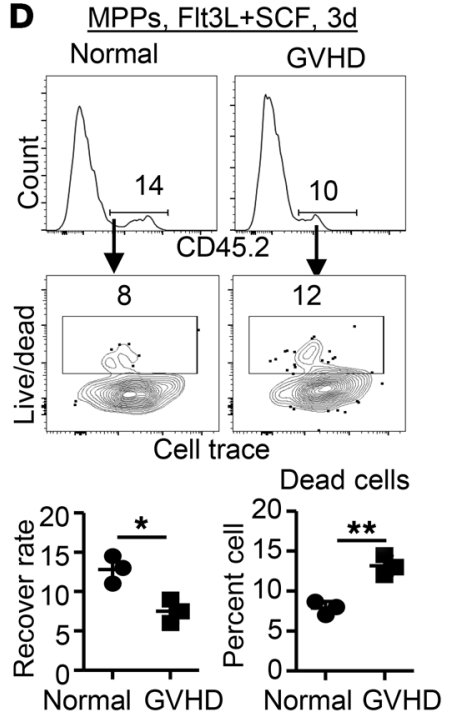

D MPPs, Flt3L+SCF, 3d

Dead cells

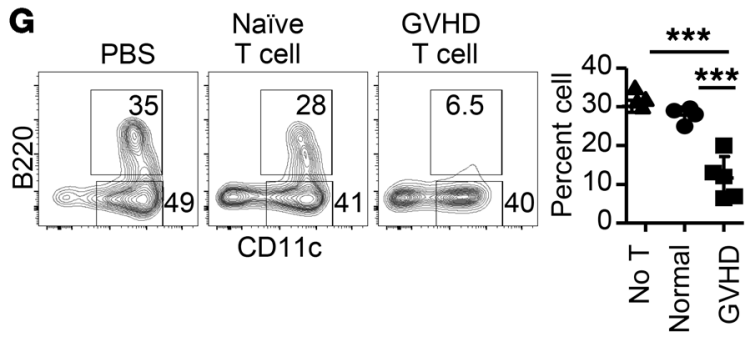

E MPPs, Flt3L+SCF, 9d

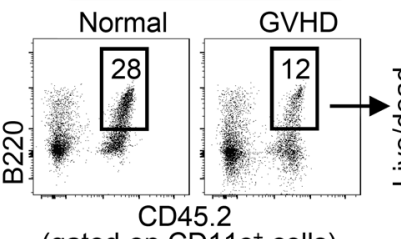

(gated on $\mathrm{CD} 11 \mathrm{c}^{+}$cells)

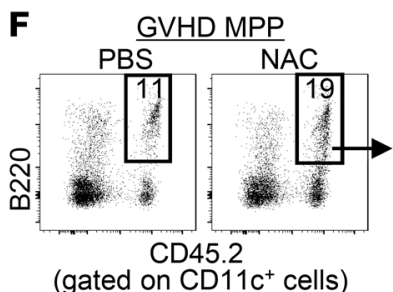

(gated on $\mathrm{CD} 11 \mathrm{c}^{+}$cells)

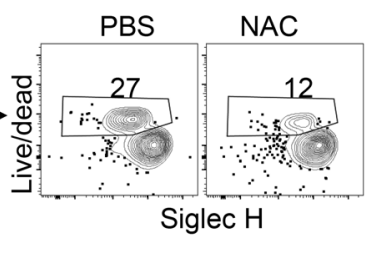

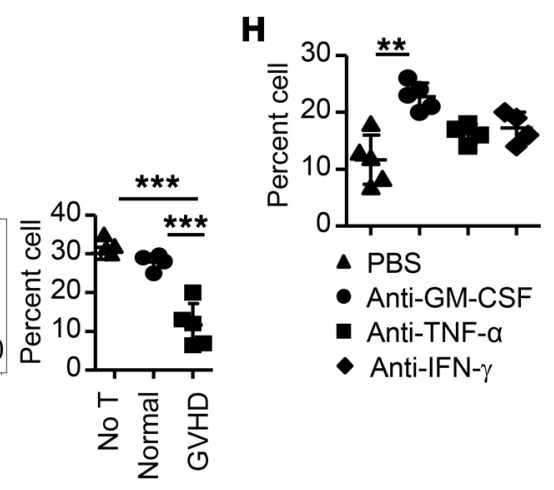

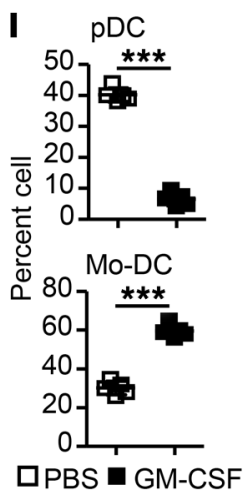

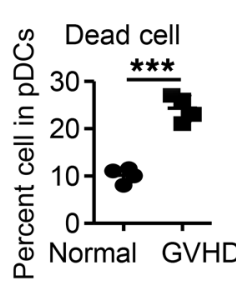
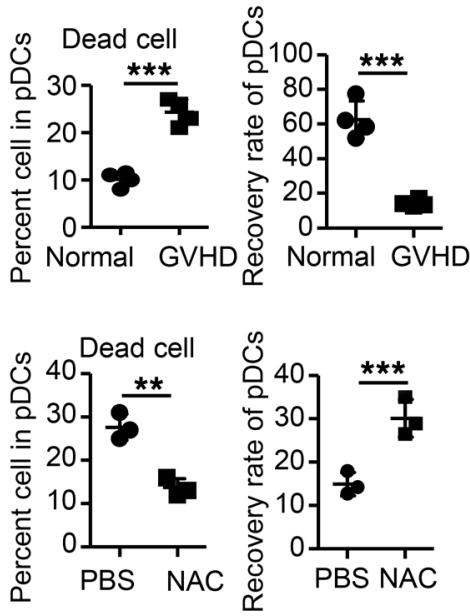
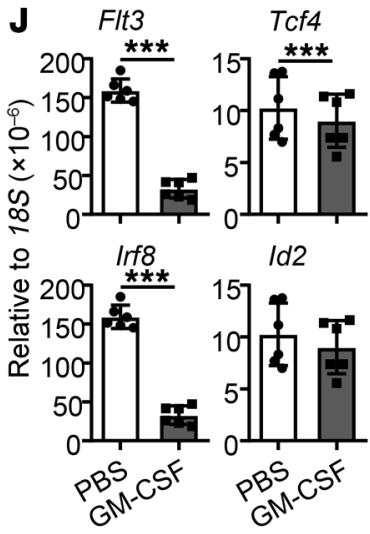

Figure 5. GVHD induces MPP loss. (A-C) GVHD in BALB/c mice was induced as described in Figure $1 \mathrm{~A}$. BM cells were isolated on day 15 after transplantation from these GVHD mice $(n=3-4)$ and activated with cytokines (i.e., Flt3L plus SCF plus GM-CSF) for 2 hours, followed by measuring p-S6K1 (A) and ROS production (B). (C) Rapamycin (1.5 mg/kg, daily, i.p.) was administered into GVHD mice from days 0 to 14 , with solvent treatment as control. MPPs were examined in these mice on day 15 after transplant. (D-F) MPPs were sorted from CVHD mice on day 15 after transplantation and labeled with CellTrace and cultured with Flt3L and SCF, with normal MPPs as controls. (D) Plots show CellTrace dilution and cell death on day 3 of culture. Graphs show the recovery rates and dead cell percentage of cultured MPPs. (E and F) On day 9 of culture, cell viability and recovery rate of pDCs were examined without (E) or with (F) NAC (500 $\mu \mathrm{M})$ for 9 days. (G) Normal c-kit ${ }^{+}$HSPCs were cultured in the upper chamber of a Transwell plate in the presence of Flt3L plus SCF. Naive T cells and GVHD T cells from normal mice and GVHD mice, respectively, were activated with anti-CD3 Ab plus anti-CD28 Ab for 3 hours and transferred into the lower chamber. Nine days later, cells were collected for pDC measurement. (H) Neutralizing Abs specific for each cytokine were added to the plate that contained c-kit+ HSPCs and activated GVHD T cells as mentioned above in G. pDC production in culture was examined 9 days later. (I and J) GM-CSF ( $5.0 \mathrm{ng} / \mathrm{mL}$ ) was added to MPP cultures stimulated with Flt3L plus SCF. (I) The frequencies of pDCs and mo-DCs were measured on day 9 . (J) Gene expression in CM-CSF-stimulated MPPs on day 3 of culture. Results shown are representative of at least 2 independent experiments. Two-group comparisons were evaluated by unpaired $t$ test (E, F, I, and J), and multiple comparisons by 1-way ANOVA with Turkey's multiple-comparison test (G) or 1-way ANOVA with Dunnett's multiple-comparison test $(\mathbf{H}) .{ }^{*} P<0.05 ;{ }^{* *} P<0.01 ;{ }^{* *} P<0.001$.

duced multiple effector cytokines such as IFN- $\gamma$, TNF- $\alpha$, and GM-CSF (Supplemental Figure 5A). It is possible that in addition to damaging the BM niche during GVHD (42), alloreactive $\mathrm{T}$ cells may directly reduce MPPs and their generation of pDCs. Indeed, GVHD T cells caused a 2.5 -fold reduction in
pDCs cultured in a Transwell plate (Figure 5G). Addition of neutralizing Ab specific for GM-CSF dramatically improved pDC generation in GVHD $\mathrm{T}$ cell cultures (Figure $5 \mathrm{H}$ and Supplemental Figure 5B). We confirmed GM-CSF's repressive effects, which induced skewed differentiation of MPPs 

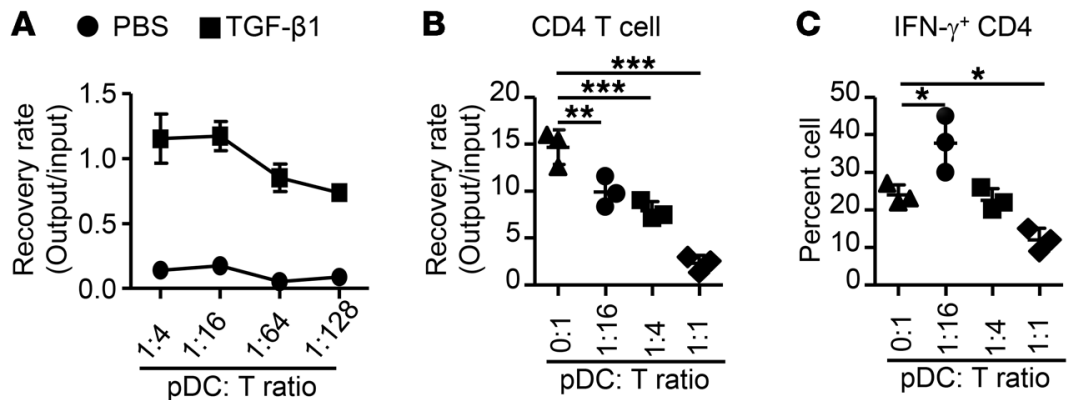

D CD8 T cell
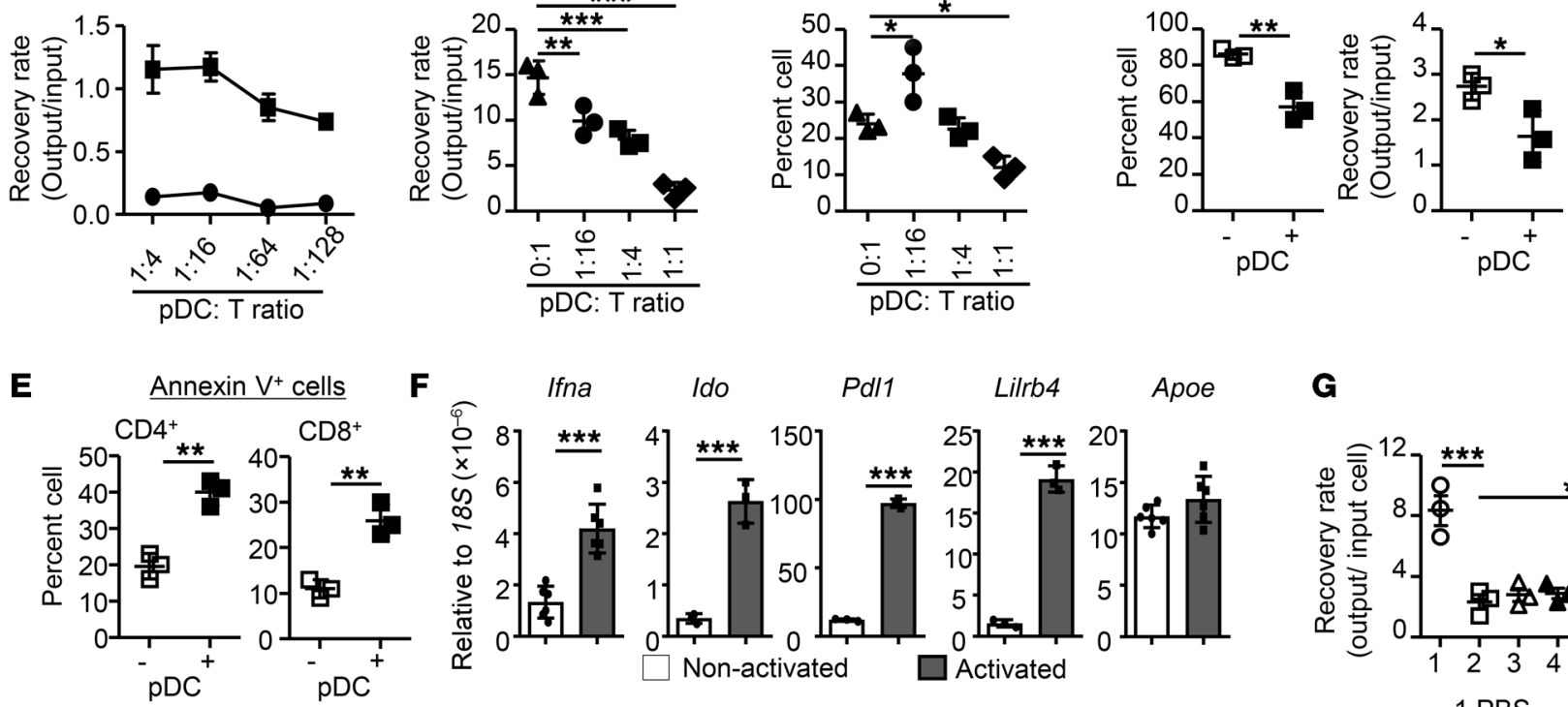

H
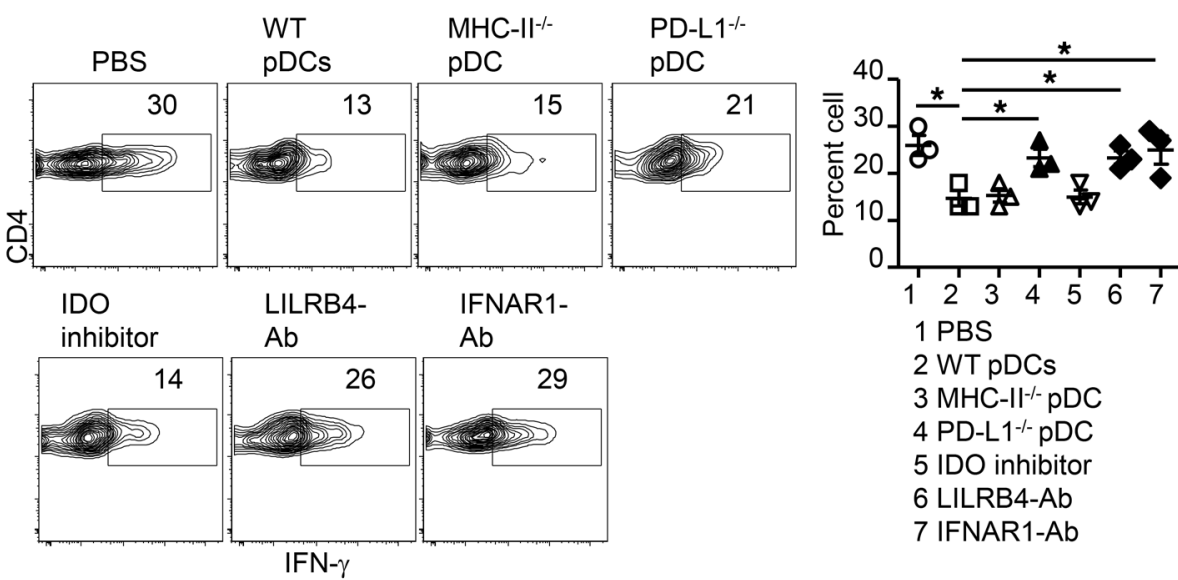

G

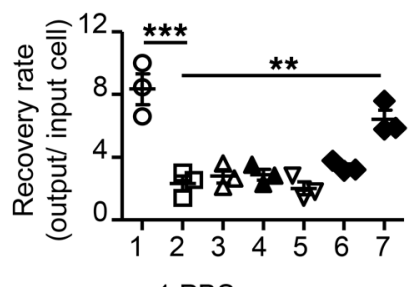

1 PBS

2 WT pDCs

$3 \mathrm{MHC}-\mathrm{II}^{-1-} \mathrm{pDC}$

4 PD-L1 ${ }^{-/-}$pDC

5 IDO inhibitor

6 LILRB4-Ab

7 IFNAR1-Ab

Figure 6. pDCs are potent suppressors of activated T cells. (A) B6 T cells were activated with anti-CD3 and anti-CD28 Abs, in the presence of B6 pDCs at various ratios of DCs/T cells, with or without TGF- $\beta 1(5 \mathrm{ng} / \mathrm{mL})$. FoxP3 ${ }^{+} C D 4^{+} T$ cells were examined on day 7 of culture. (B-D) Naive CD4 ${ }^{+}$or CD8 ${ }^{+} T$ cells were labeled with CellTrace Violet and activated by anti-CD3/anti-CD28 Abs in the presence of different doses of B6 pDCs. Seven days later, cells were counted to examine the recovery rate of activated $C D 4^{+} T$ cells (B), IFN- $\gamma$ expression by $C D 4^{+} T$ cells (C), and recovery rate of activated CD8 ${ }^{+} T$ cells $(D)$. (E) Graphs show T cells undergoing apoptosis upon annexin $V$ staining. (F) Real-time RT-PCR analysis of genes in ex vivo generated B6 pDCs before and after activation of TLR4 and TLR7/8. (G and H) B6 T cells were activated by anti-CD3/anti-CD28 Abs with or without addition of syngeneic pDCs. Blocking Abs or inhibitors were added in different groups. Seven days later, cells were counted (G) and tested for IFN- $\gamma$ production (H). Data shown are mean \pm SD. Results shown are representative of 2 to 3 independent experiments. Multiple comparisons were evaluated by 1-way ANOVA with Dunnett's multiple-comparison test (B, C, G, and $\mathbf{H}$ ), and 2-group comparisons by unpaired $t$ test (D-F). ${ }^{*} P<0.05 ;{ }^{* *} P<0.01 ;{ }^{* *} P<0.001$.

into cDCs (Figure 5I and Supplemental Figure 5C). This was accompanied by decreases in Flt3 and transcription factor (e.g., Irf8, Tcf4) gene expression (Figure 5J), which are critical for pDC development (4).

Previous studies have suggested that GVHD prevents the maturation of pDCs while promoting the generation of pre-DCs (14). Compared with MPPs, pre-DCs significantly upregulated the expression of Tcf4 (Supplemental Figure 5D), confirming their commitment to the pDC lineage. Addition of GM-CSF affected neither the generation of mature pDCs nor the upregulation of MHC-II (Supplemental Figure 5, E and F). Collectively, these results show that alloreactive $\mathrm{T}$ cells impair MPP differentiation into pDCs but do not stymie pDC maturation.
Donor-type pDCs directly repress alloreactive $T$ cell responses in vitro. Although pDCs are known to be tolerogenic $(24,25)$, the precise role of pDCs in repressing alloimmunity remains poorly defined. pDCs have been shown to promote Treg expansion and function $(4,25,26)$. However, we found that donor-type pDCs alone failed to expand autologous $\mathrm{CD} 4^{+}$Tregs in cultures (Figure 6A and Supplemental Figure 6A). Addition of pDCs to TGF- $\beta 1^{-}$ induced $\mathrm{CD} 4^{+} \mathrm{T}$ cell cultures moderately enhanced Treg numbers (Figure 6A), suggesting that pDCs likely potentiate TGF- $\beta 1$ induction of Tregs. To specifically examine whether pDCs augment the generation of inducible Tregs (iTregs), we isolated $\mathrm{GFP}^{-} \mathrm{CD} 4^{+}$ $\mathrm{T}$ cells from Foxp $3^{\text {EGFP }} \mathrm{B} 6$ mice and cultured them with B6 pDCs and TGF- $\beta 1$ (Supplemental Figure 6, B and C). pDCs promoted 
A

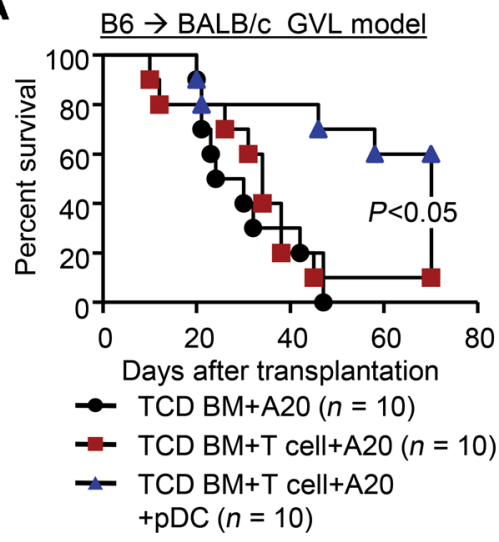

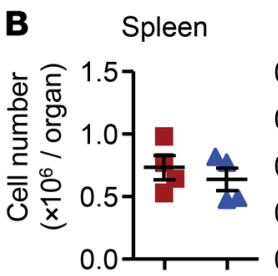
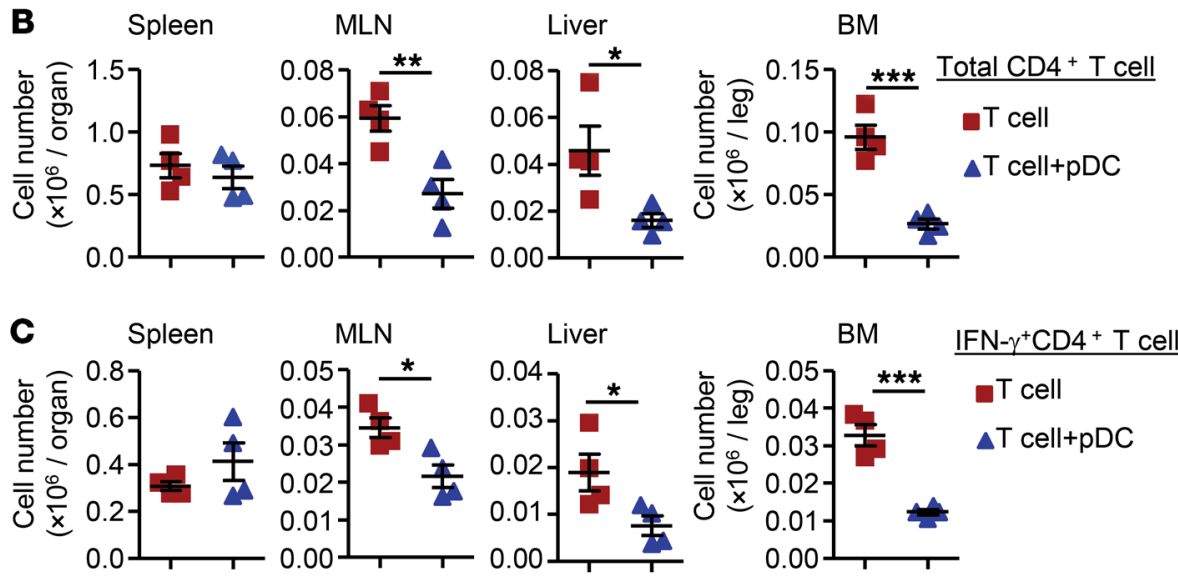

E

D

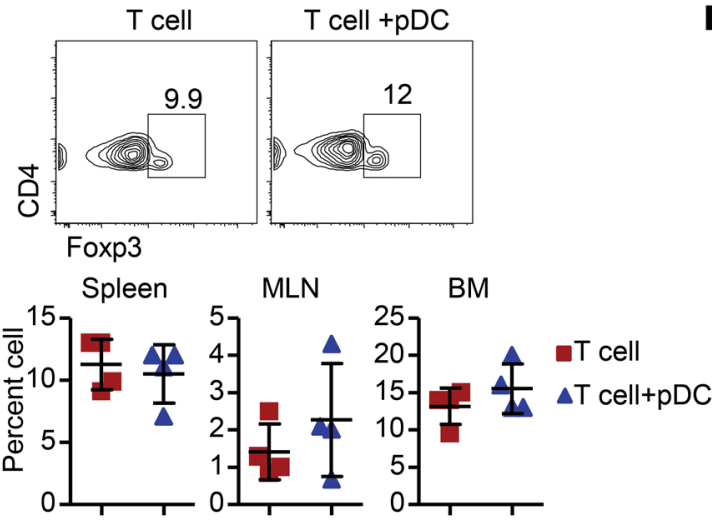

B6 T cells $+\mathrm{pDCs}$ $\left(\mathrm{CD} 45.2^{+} \mathrm{H} 2 \mathrm{~b}^{+}\right)$ $+$

B6/SJL TCD-BM (CD45. $\left.1^{+}+\mathrm{H}^{\mathrm{b}} \mathrm{b}^{+}\right)$

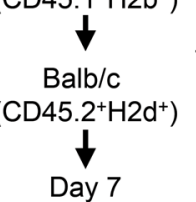

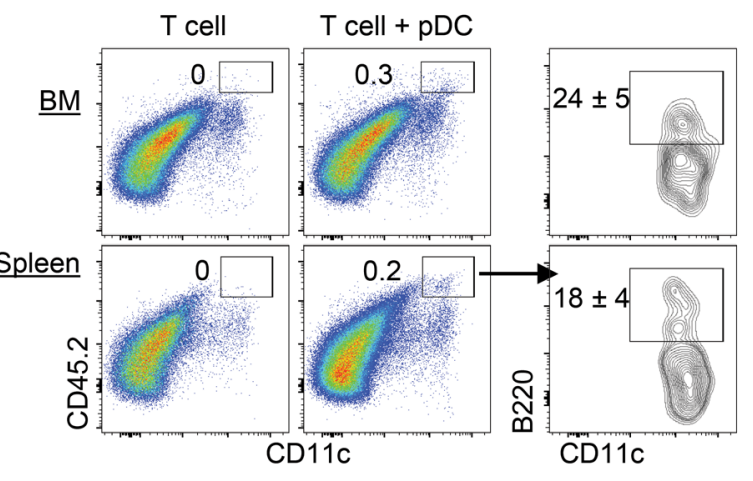

$\mathbf{F}$
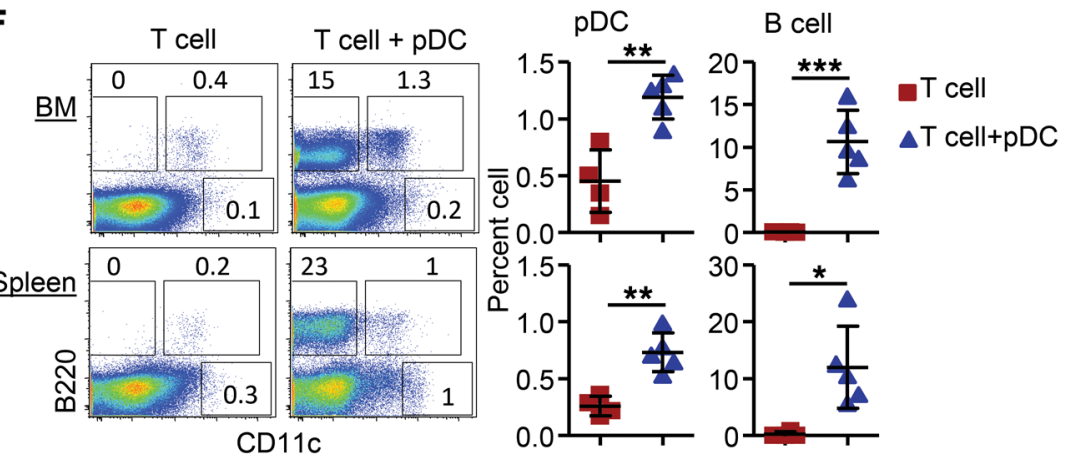

Figure 7. Transfer of donor-type pDCs preserves GVL activity while reducing alloreactive $T$ cell responses in mice. (A) BALB/c recipients of B6 T cells and TCD-BM were challenged with A20 leukemia cells $\left(1 \times 10^{6}\right)$ and treated with or without B6 pDCs $\left(1 \times 10^{6} /\right.$ day on days 0 , 1 , and 2$)$. Survival was monitored. (BD) B6 TCD-BM plus CD4+ ${ }^{+}$cells were transferred to lethally irradiated BALB/c mice ( $n=4$ per group), followed with or without injection of B6 pDCs $\left(1 \times 10^{6}\right)$ on days 0,1 , and 2. (B) Donor cells were collected on day 7 to count donor CD4+ $T$ cells, (C) test cytokine production, and (D) examine the generation of CD4 Tregs. MLN, mediastinal lymph nodes. (E) B6/SJL (CD45.1 $1^{+}$TCD-BM plus CD4+ $T$ cells were transferred to lethally irradiated BALB/C mice ( $n=4$ per group), followed with or without injection of B6 pDCs (CD45.2+) on days 0, 1, and 2. Donor cells were collected on day 7 for infused-pDC analysis. (F) Thirty-four days after transplantation, donor cells were collected to analyze donor pDC reconstitution ( $n=3$ per group). Survival comparisons made by log-rank test (A), and 2-group comparisons by unpaired $t$ test (B-D and $\mathbf{F}$ ). Data shown are mean \pm SD. Results shown in $\mathbf{A}-\mathbf{D}$ and $\mathbf{F}$ are representative of 2 independent experiments. ${ }^{*} P<0.05$; ${ }^{* *} P<0.01$; ${ }^{* *} P<0.001$.

the generation of iTregs only in the presence of TGF- $\beta 1$ (Supplemental Figure 6, B and C). Thus, pDCs induce iTregs in a TGF- $\beta 1$ dependent manner.

Interestingly, mature pDCs repressed the proliferation of TCR-activated autologous $\mathrm{CD} 4^{+} \mathrm{T}$ cells, decreasing numbers of IFN- $\gamma$-producing effectors in a dose-dependent way (Figure 6, B and C, and Supplemental Figure 7, A and B). Mature pDCs also moderately but significantly repressed autologous $\mathrm{CD}^{+} \mathrm{T}$ cell proliferation (Figure 6D). Donor-type pDCs caused significant increases in apoptotic cells in both TCR-activated $\mathrm{CD} 4^{+}$and $\mathrm{CD} 8^{+}$ $\mathrm{T}$ cells (Figure 6E and Supplemental Figure 7, $\mathrm{C}$ and D), but did not affect $\mathrm{T}$ cell activation (Supplemental Figure 7, $\mathrm{E}$ and $\mathrm{F}$ ).

Activation of mature pDCs induced high levels of immunosuppressive molecules $(45,46)$, including PD-L1, IDO, and LILRB4 (Figure 6F and Supplemental Figure 7G). Furthermore, these pDCs produced high levels of APOE (Figure 6F), which can acti- 
vate LILRB4 in monocytes and macrophages (46). These data indicate that activated pDCs can suppress $\mathrm{T}$ cell responses through multiple suppressive mechanisms. Notably, genetic deletion of either PD-L1 or MHC-II in pDCs did not affect their suppressive capabilities (Figure 6G). IDO blockade did not affect pDCs' repressive effects (Figure 6G). Addition of neutralizing Ab specific for LILRB4 failed to improve the expansion of pDC-treated T cells in culture (Figure 6G). Surprisingly, blockade of IFNAR1 using its neutralizing Ab largely abrogated pDCs' repressive effects (Figure $6 G)$. We further examined the impact of these molecules on effector differentiation of pDC-treated syngeneic $\mathrm{T}$ cells. Individual blockade of IFNAR1, PD-L1, and LILRB4 significantly increased the frequency of IFN- $\gamma$-producing cells compared with the untreated control (Figure 6H).

Altogether, our data indicate that pDCs modulate alloimmunity in a complex manner; mature pDCs repress TCR-activated T cell proliferation and expansion via a type I IFN signaling-dependent mechanism. Furthermore, we found that PD-L1 and LILRB4 may also contribute to $\mathrm{pDC}$ repression of effector differentiation.

Transfer of donor-type pDCs preserves graft-versus-leukemia activity while reducing alloreactive $T$ cell responses in mice. Finally, we assessed whether donor-type pDCs can preserve graft-versus-leukemia (GVL) effects in mice after allo-HSCT using the B6 $\rightarrow$ BALB/C mouse model. The transfer of donor $\mathrm{pDCs}$ preserved antileukemic activity but reduced GVHD, leading to a significantly improved overall survival rate for leukemic mice undergoing allo-HSCT (Figure 7A). To examine the underlying mechanism, we adoptively transferred donor-type pDCs (B6 origin) into allo-HSCT BALB/c recipients on days 0,1 , and 2 after transplantation and isolated donor T cells on day 7. Untreated allo-HSCT BALB/c recipients were used as controls. Donor-type pDCs reduced numbers of alloreactive $\mathrm{CD} 4^{+} \mathrm{T}$ cells and IFN- $\gamma$-producing effector $\mathrm{T}$ cells in the BM, mesenteric lymph nodes, and liver (Figure 7, B and C), but did not significantly affect Foxp $3^{+}$Tregs (Figure 7D). However, the transfer of donor-type pDCs did not markedly decrease the frequency of IFN- $\gamma$-producing alloreactive $\mathrm{CD}^{+}{ }^{+} \mathrm{T}$ cells in the liver, spleen, and BM (Supplemental Figure 8, A and B). A substantial proportion of donor alloreactive $\mathrm{T}$ cells retained their ability to produce IFN- $\gamma$ (Figure 7C), a key cytokine that mediates GVL effects (47-49).

We used 2 additional mouse models to test pDC applicability to GVL-effect preservation. In the $\mathrm{B} 6 \rightarrow \mathrm{BALB} / \mathrm{c}$ mouse model challenged using P815 mastocytoma cells, all mice receiving T cell-depleted or T cell-replete BM died from tumors by day 21 after transplantation (Supplemental Figure 9, A and B). In contrast, transfer of pDCs with $\mathrm{CD} 4^{+} \mathrm{T}$ cells protected 3 of 8 recipients against the P815 tumors (Supplemental Figure 9, A and B). In the $\mathrm{BALB} / \mathrm{c} \rightarrow \mathrm{B} 6$ transplant model challenged by MBL2 acute myeloid leukemia (AML) cells $\left(\mathrm{H}-2^{\mathrm{b}}\right)$, all $\mathrm{B} 6$ mice receiving TCD-BM died from leukemia. To induce GVL activity in mice without causing severe GVHD in these B6 mice, we transferred low doses of donor $\mathrm{CD}^{+}{ }^{+}$and $\mathrm{CD} 8^{+} \mathrm{T}$ cells $\left(0.5 \times 10^{6}\right.$ cells/mouse $)$. This dose of donor $\mathrm{T}$ cells failed to induce lethal GVHD in recipients but protected them from leukemia (Supplemental Figure 9, C and D). Donor pDC therapy did not significantly impair antileukemic activity, with 4 of 5 of them surviving without leukemia and GVHD (Supplemental Figure 9, C and D). Using the MLR assay as an indicator of allogeneic $\mathrm{T}$ cell response, we showed that donor pDCs dramat- ically suppressed the responses of both $\mathrm{CD}^{+}$and $\mathrm{CD} 8^{+} \mathrm{T}$ cells to allogeneic DCs, with decreased numbers of $\mathrm{T}$ cells producing IFN- $\gamma$ (Supplemental Figure 9, E and F). The numbers of P815 cells and MBL2 cells, which we used for inducing leukemia and testing the GVL effects (Supplemental Figure 9, A-D), have been previously established by several groups including ours (32, 50-52). All these results indicate that donor pDC therapy can largely preserve donor T cells' antileukemic activity. This is further supported by others' observations that pDC treatment does not compromise GVL effects in other mouse models of human allo-HSCT $(28,53)$.

Interestingly, the infused mature donor $\mathrm{pDCs}$ were still detected in the spleen and BM in allo-HSCT mice 7 days after transplant (Figure 7E). Furthermore, the transfer of donor pDCs early after allo-HSCT significantly increased the de novo generation of donor pDCs later after allo-HSCT (Figure 7F). Also notable is the observation that donor pDC treatment significantly improved the reconstitution of $\mathrm{B} 22 \mathrm{O}^{+} \mathrm{B}$ cells in the $\mathrm{BM}$ and spleen (Figure $7 \mathrm{~F}$ ); this finding is important because it is known that impaired $\mathrm{B}$ cell reconstitution reflects a GVHD-damaged BM niche (42). Altogether, these data suggest that short-term treatment with donor pDCs may result in improved donor $\mathrm{pDC}$ reconstitution, leading to a long-term positive impact on the prevention of severe GVHD. In particular, the transfer of donor pDCs protects against $\mathrm{GVH}$ reaction-mediated damage to the $\mathrm{BM}$ niches.

Altogether, our findings indicate that alloreactive $\mathrm{T}$ cell responses and GVHD cause the loss of MPPs and subvert MPP production of $\mathrm{pDCs}$, leading to donor $\mathrm{pDC}$ defects. $\mathrm{pDC}$ produce multiple immunosuppressive molecules, can directly repress proliferation and expansion of effector $\mathrm{T}$ cells, and possess a potent ability to repress GVHD. Thus, it is likely that the improved recovery of donor pDCs early after allo-HSCT can break a feed-forward GVHD cascade.

\section{Discussion}

Our studies uncover the mechanism by which donor pDCs fail to reconstitute during GVHD and the crucial role donor pDCs play in repressing the disease. MPPs are critical for sustaining pDCs, but are depleted during GVHD, the disease having the largest impact on Flt $3^{\text {hi }}$ MPPs. GM-CSF derived from alloreactive T cells subverted MPP production of pDCs. Combined, alloreactive T cell responses and GVHD cause donor pDC reconstitution to fail. We observed that pDCs suppressed the proliferation and expansion of activated autologous $\mathrm{CD} 4^{+} \mathrm{T}$ cells via a type I IFN signaling-dependent mechanism. Enhanced recovery of donor pDCs upon adoptive transfer early after allogeneic HSC transplantation repressed GVHD and restored de novo generation of donor pDCs in recipient mice. pDCs suppressed the proliferation and expansion of activated autologous $\mathrm{T}$ cells via a type I IFN signalingdependent mechanism. They also produced PD-L1 and LILRB4 to inhibit T cell production of IFN- $\gamma$. We thus demonstrated that GVHD impairs the reconstitution of tolerogenic donor pDCs by depleting DC progenitors rather than by preventing pDC maturation. A short-term treatment with donor pDCs results in long-term GVHD repression.

The dysregulated reconstitution of functional donor DCs is a known complication of GVHD (12-14, 27, 32, 54). Because alloreactive $\mathrm{T}$ cells produce multiple inflammatory cytokines and medi- 
ate cytotoxicity against host tissue damage, many studies have assumed that impaired DC reconstitution is attributable to the disruption of the DC maturation process $(8,13,14)$. Indeed, DCs have a short life span and rapidly diminish during inflammation (4). A previous study suggested that GVHD mice were depleted of donor mature pDCs (14). Donor-derived CD11 $\mathrm{c}^{\text {lo }}$ PDCA- $1^{+}$pre-DCs transiently appeared between 7 and 14 days after transplantation, but rapidly diminished upon GVHD onset (14). Intriguingly, although these "pre-DCs" could become cDCs and pDCs upon transfer into syngeneic mice, they lacked B220 and IFN- $\alpha$ expression and produced high levels of MHC-II and CD86 (14). Thus, these donor "pre-DCs" generated during GVHD possess the phenotype of activated mature DCs (1-3). However, results from this study do not explain how GVHD causes rapid diminishment of these "preDCs" and pDC defects (14). Our data indicate that the depletion of MPPs and impairment of MPP function are likely more significant contributors to dysregulated donor DC reconstitution than is the disruption of DC maturation. MPPs may represent an effective target for the support of pDC reconstitution to control GVHD.

Our findings suggest that decreases in MPPs were associated with decreased MPP generation and maintenance during GVHD. HSCs lack cell-surface Flt3 expression, differentiating into Flt3 ${ }^{+}$ MPPs that give rise to CDPs and other hematopoietic cell lineages $(3,4)$. GVHD led to significantly fewer HSCs in the BM, which likely contributed to an overall decrease in MPP generation. In addition, during GVHD, MPPs had hyperactivated mTOR and increased oxidative cell death, leading to impaired MPP maintenance. This is consistent with previous observations that sustained mTOR activity results in defects in HSPC function (19). Thus, GVHD-mediated loss of functional MPPs is the culmination of several discrete processes and outcomes, including decreased numbers of donor HSCs that could give rise to MPPs and increased death of proliferative MPPs.

Inhibition of MPP differentiation into pDCs during GVHD may represent another major contributor to impaired donor $\mathrm{pDC}$ reconstitution during GVHD. Flt3L is crucial for pDC development, whereas GM-CSF induces the generation of inflammatory DCs. We found that alloreactive effector $\mathrm{T}$ cells produced high levels of GM-CSF. Blockade of GM-CSF using neutralizing Ab significantly reduced alloreactive $\mathrm{T}$ cell-mediated inhibition of $\mathrm{pDC}$ production in culture. Recent studies have demonstrated that the alloreactive effector $\mathrm{T}$ cell subset that produces GM-CSF is critical for promoting intestinal GVHD (55). GM-CSF drives GVHD pathology by recruiting and activating antigen-presenting cells to produce inflammatory mediators (56). Furthermore, donor T cells lacking GM-CSF or subjected to GM-CSF blockade resulted in significantly lower GVHD incidence in allogeneic BMT recipient mice (57). In patients, an elevated number of GM-CSF-producing $\mathrm{T}$ cells is associated with severe GVHD (56). GM-CSF inhibited the expression of Flt3 in MPPs and their upregulation of transcription factors critical for pDC development (i.e., Irf8 and Tcf4). It is possible that alloreactive $\mathrm{T}$ cells produced GM-CSF to break pDC-mediated tolerance against host tissues, thereby invigorating GVHD's inflammatory cascade.

pDC repression of alloreactive $\mathrm{T}$ cell responses likely involves a complex mechanism. Many studies have revealed the importance of pDCs in promoting Treg expansion and function. For example, CCR9 $9^{+} \mathrm{pDCs}$ promote expansion and function of allogeneic Foxp $3^{+} \mathrm{CD}^{+}$Tregs in culture (26). pDCs produce high levels of IDO to promote Treg expansion (28). In contrast, in the setting of T cells incubated with autologous pDCs, we found that although pDCs synergized with TGF- $\beta 1$ enhanced the expansion of syngeneic Tregs, the pDCs on their own had a limited ability to induce the expansion of autologous Tregs in vitro. Instead, pDCs had a potent ability to directly repress proliferation and expansion of TCR-activated autologous T cells. Furthermore, administration of donor-type pDCs also repressed expansion of host-reactive donor $\mathrm{T}$ cells in GVHD mice. Surprisingly, blockade of IFNAR1 significantly decreased pDCs' repressive effects. This suggests that IFN- $\alpha$ may be an important factor mediating $\mathrm{pDC}$ repression of $\mathrm{T}$ cell responses. Previous studies have shown that the administration of exogenous IFN- $\alpha$ during conditioning alleviated GVHD by reducing $\mathrm{T}$ cell proliferation and effector differentiation (58). Given that pDCs are typically significant IFN- $\alpha$ producers, it is likely that donor-type pDCs control GVHD via IFN- $\alpha$-mediated repression of alloreactive $\mathrm{T}$ cell responses.

However, data from our and other studies indicate that pDCs express many other immunosuppressive molecules (e.g., PD-L1, IDO, LILRB4, APOE, etc.) $(4,27,28,30)$. Although we did not demonstrate the effects of these individual molecules on mediating $\mathrm{pDC}$ repression of $\mathrm{T}$ cell proliferation in culture, we do not rule out the possibility that $\mathrm{pDCs}$ require these molecules to repress GVHD in vivo. In fact, we found that PD-L1 and LILRB4 were engaged in $\mathrm{pDC}$-mediated repression of IFN- $\gamma$ production by TCR-activated T cells. Thus, it will be important to investigate the precise role of these molecules in $\mathrm{pDC}$ repression of antigen-driven $\mathrm{T}$ cell responses in vivo.

Our findings illuminate a what we believe is a new method of regulating donor T cells' GVHD and GVL effects via the transfer of activated donor pDCs. We observed that donor pDC therapy significantly reduced the total number of alloreactive $\mathrm{T}$ cells but allowed the persistence of a sizable number of IFN- $\gamma$-secreting alloreactive $\mathrm{T}$ cells. IFN- $\gamma$ plays paradoxical roles in regulating GVH reactivity against lymphohematopoietic cells versus nonhematopoietic tissues (59-63). IFN- $\gamma$ is known to prevent severe GVHD early after allo-HSCT in mice and repress damage to the colon, skin, and lung $(47,59-61,64)$. For example, donor T cellderived IFN- $\gamma$ upregulated the expression of IDO and PD-L1 in the local tissues, which in turn diminished $\mathrm{T}$ cell proliferation and inflammation $(47,63)$. Thus, having few IFN- $\gamma$-producing alloreactive $\mathrm{T}$ cells in the host hematolymphoid compartment may be sufficient to mediate a potent GVL effect $(47,48,65)$. Altogether, our findings significantly further our understanding of the role donor pDCs play in clinical allo-HSCT. Novel strategies for donor pDC-based therapy may effectively regulate donor $\mathrm{T}$ cell function to optimize allo-HSCT-related GVL activity in patients with hematological malignancies.

Our studies together with others $(28,30,53)$ suggest that Flt3L improves the ability of pDCs to prevent and treat GVHD. Clinically relevant approaches to produce adequate amounts of $\mathrm{pDCs}$ remain a bottleneck that limits pDC therapy feasibility for GVHD prevention and treatment. Elegant studies by Waller's group suggest that in vivo administration of Flt3L represents a promising strategy to increase the number of pDCs (53). Donor mice receiving 2 doses 
of Flt3L treatment had a 5-fold increase in pDC content without significant impact on HSCs. Like untreated pDCs from normal mice, these Flt3L-stimulated pDCs reduced GVHD and preserved GVL, significantly improving the survival of mice with leukemia receiving allo-HSCT. This beneficial effect of donor pDCs has also been observed in other studies of both murine and human systems $(28,30)$. Flt3L in culture induces pDCs resembling those that differentiate in vivo in animals treated with FLT3L $(4,66)$. Notably, human pDCs derived from Flt3L-treated donors have similar gene expression profiles to Flt3L-induced murine pDCs (53). Herein we demonstrate that adequate numbers of pDCs can be generated from Flt3L-stimulated cultures. Since the production of pDCs using cultured HSPCs allows the addition of different growth factors, we propose that it offers a unique opportunity to optimize the quantity and quality of producing immune-regulatory pDCs to serve as a template potentially for future manufacturing protocols. Taken together, these data suggest translation of pDC therapy to allo-HSCT patients is a promising strategy.

In summary, our findings open new avenues for investigation of the molecular mechanisms through which GVHD causes dysregulated reconstitution of donor DCs. We also provide clear evidence that the transfer of donor-type pDCs may represent an effective and novel cellular therapy for GVHD prevention. However, challenges in producing adequate amounts of donor-type pDCs remain a bottleneck limiting the applicability of donor pDC therapy for GVHD inhibition. Further studies will involve the optimization of tolerogenic pDC production for therapeutic purposes. Since HSPCs' regenerative capacity is impaired during chronic infection, autoimmunity, and cancer (16-19), and since pDCs play crucial roles in protection against viral infections, our studies' impact extends beyond allo-HSCT and may have broad implications for many other diseases.

\section{Methods}

Healthy donors and patients. $\mathrm{PB}$ and BM from deidentified healthy donors and patients undergoing allo-HSCT were collected in this study after obtaining informed consent. The characteristics of the allogeneic HSCT recipients are summarized in Supplemental Tables 1-3. PB and $\mathrm{BM}$ were obtained at the time of GVHD onset (on average between days 21 and 70 after transplantation). Some BM samples were obtained from healthy adult donors who were providing BM for allo-HSCT.

Mice. C57BL/6 (B6, H-2 $\left.{ }^{\mathrm{b}}, \mathrm{CD} 45.2^{+}\right), \mathrm{B} 6 / \mathrm{SJL}\left(\mathrm{H}-2^{\mathrm{b}}, \mathrm{CD} 45.1^{+}\right)$BAL$\mathrm{B} / \mathrm{c}\left(\mathrm{H}-2^{\mathrm{d}}\right)$, and $\mathrm{B} 6 \mathrm{xDBA} / 2 \mathrm{~F} 1\left(\mathrm{BDF} 1, \mathrm{H}-2^{\mathrm{b} / \mathrm{d}}\right)$ mice were purchased from The Jackson Laboratory. Abb (H2-Ab1) mutant B6 mice that do not express MHC class II molecules ( $\mathrm{MHC}-\mathrm{II}^{-/}$) were purchased from Taconic. Foxp $3^{E G F P}$ mice were purchased from The Jackson Laboratory.

$G V H D$ and GVL response. Mice underwent BM transplantation as described previously (32). GVHD scores and severity were graded according clinical parameters and histopathological analysis. To induce GVHD, we irradiated BALB/c recipients with 850 cGy from an x-ray source followed by transplantation with donor B6 TCD-BM with or without $\mathrm{B} 6 \mathrm{CD} 4^{+} \mathrm{T}$ cells. $\mathrm{B} 6 \mathrm{TCD}-\mathrm{BM}$ with $\mathrm{CD}^{+}$and $\mathrm{CD}^{+}{ }^{+} \mathrm{T}$ cells were transferred into lethally irradiated BDF1 mice (11.5 Gy). In GVL experiments, we injected A20 $0^{\mathrm{TGL}}$ cells $\left(1 \times 10^{6}\right.$ cells/mouse) or p815 $\left(3 \times 10^{3}\right)$ or MBL2 cells $\left(3 \times 10^{4}\right) 2$ hours before HSCT (which reflects residual disease in human transplant recipients) and monitored leukemic growth using bioluminescence imaging as described previously $(67,68)$.
DC production. Mouse BM was cultured in RPMI 1640 medium (Gibco) containing 10\% fetal bovine serum (Gibco), recombinant mouse Flt3L (50 ng/mL), and SCF (10 ng/mL) (Shenandoah Biotechnology) for 9 days, as described previously $(32,69)$. To induce DC activation, immature DCs were incubated with LPS $(100 \mathrm{ng} / \mathrm{mL}$ ), R848 (Resiquimod, $100 \mathrm{ng} / \mathrm{mL}$ ), or CpG (ODN 1585, $1 \mu \mathrm{M}$ ) (InvivoGen) overnight, which activated Toll-like receptor 4 (TLR4), TLR7/8, or TLR9 signaling, respectively. Cells were harvested for experiments. pDCs were sorted to isolate $\mathrm{CD} 11 \mathrm{c}^{+} \mathrm{B} 22 \mathrm{O}^{+}$cells using FACS and magnetic beads (Miltenyi Biotec).

Abs, flow cytometry analysis, and cell sorting. All Abs used for immunofluorescence staining were purchased from eBioscience, BioLegend, or BD Biosciences. Magnetic microbead-conjugated Abs and streptavidin were purchased from Miltenyi Biotec. The antibodies are listed in Supplemental Table 4. Recombinant human IL-2 protein was purchased from R\&D Systems. Recombinant mouse Flt3L and mouse SCF were purchased from Shenandoah Biotech. Cells were stained with appropriate concentrations of mAbs. Dead cells were excluded using Fixable Viability Dye from eBioscience. Flow cytometry analyses were performed using LSRII (BD Biosciences) and FACSCanto cytometers (BD Biosciences). Cell were sorted on a FACSAria II (BD Biosciences).

Human DCs were characterized as negative for lineage markers (CD3, CD14, CD15, CD16, CD19, CD20, and CD56) and positive for HLA-DR as described previously (36). Among these human DCs, $\mathrm{CD}_{123^{+}}$cells were defined as $\mathrm{pDCs}$ and $\mathrm{CD} 1 \mathrm{c}^{+} \mathrm{CD} 123^{-}$cells were defined as cDCs. Human HSPCs were gated as CD34 $4^{+}$. Human MPPs were characterized as $\mathrm{CD}^{+} 4^{+} \mathrm{CD} 38^{-} \mathrm{CD}^{-} 0^{-} \mathrm{CD} 45 \mathrm{RA}^{-}$. Human CDPs were characterized as $\mathrm{CD} 34^{+} \mathrm{CD} 38^{-} \mathrm{CD} 10^{-} \mathrm{CD} 45 \mathrm{RA}^{+} \mathrm{CD} 123^{+} \mathrm{CD} 115^{-}$.

Mouse pDCs were characterized as $\mathrm{CD} 11 \mathrm{c}^{+} \mathrm{B} 22 \mathrm{O}^{+}$Siglec- $\mathrm{H}^{+}$cells and

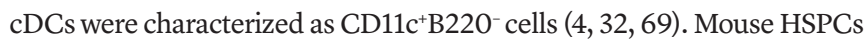
were characterized as negative for lineage markers (CD3, CD11b, Gr1, Ter119, and B220) and positive for c-kit. LT-HSCs were characterized as $\mathrm{Lin}^{-} \mathrm{Sca1}^{+} \mathrm{c}-\mathrm{kit}^{\mathrm{hi}} \mathrm{Flt3} 3^{-} \mathrm{CD} 48^{-} \mathrm{CD} 150^{+}$. MPPs were characterized as $\mathrm{Lin}^{-}$ $\mathrm{Sca}^{+} \mathrm{c}-\mathrm{kit}^{\mathrm{hi}} \mathrm{Flt}^{+}{ }^{+}$. CDPs were characterized as $\mathrm{Lin}^{-} \mathrm{Sca} 1^{+} \mathrm{c}-\mathrm{kit}^{\mathrm{int}} \mathrm{Flt} 3^{+}$.

$\mathrm{T}$ cell preparations. TCD-BM was prepared by depleting $\mathrm{T}$ cells with microbead-conjugated anti-CD4/anti-CD8 Abs. Naive T cells were isolated from spleens and lymph nodes using microbead-conjugated Abs (MiniMACS; Miltenyi Biotec). Purity was consistently greater than $92 \%$.

RNA sequencing analysis. RNA sequencing (RNA-seq) was carried out at the sequencing core at the Fox Chase Cancer Center. Transcriptome analysis was performed on RNA isolated from fresh sorted Lin $^{-}$Sca1 ${ }^{+}$c-kit ${ }^{\text {hi }} \mathrm{CD} 135^{-}$cells from healthy mice or mice experiencing GVHD. Total RNA was isolated from cells using an RNeasy Mini Kit (QIAGEN) and RNA-seq libraries were prepared using SureSelect RNA Library Preparation kits (Agilent Technologies). Samples were run on a HiSeq 2000 sequencing system (Illumina), and at least $37.5 \times 10^{6}$ single-end reads were obtained per sample. Expression was evaluated by determining the fragment per kilobase per million reads values. Using 1-way ANOVA, we selected transcripts with $P<0.01$ and $q<0.01$ for comparing paired groups and with at least a 1.5-fold difference from the means for the paired groups. Raw sequence reads were aligned to the mouse genome (mm10) using the Tophat algorithm; the Cufflinks algorithm was implemented to assemble transcripts and estimate their abundance. Cuffdiff was used to statistically assess expression changes in quantified genes in different conditions. FDR $<5 \%$ and FC $\geq 2$ were used as cutoffs to identify significantly changed genes, as described previously (70). RNA-seq data were deposited in the NCBI's Gene Expression Omnibus database with accession number GSE143788. 
Real-time RT-PCR. Total RNA was extracted from sorted DCs and HSPCs using an RNeasy Micro Kit (QIAGEN) and reverse transcribed with a SuperScript VILO cDNA Synthesis Kit (Invitrogen). cDNA was quantified through quantitative real-time polymerase chain reaction (PCR) using SYBR Green PCR mix (Applied Biosystems) on an Applied Biosystems PCR system. Thermocycler conditions included an initial hold period at $95^{\circ} \mathrm{C}$ for 2 minutes; this was followed by a 3-step PCR program, as follows: $95^{\circ} \mathrm{C}$ for 30 seconds, $55^{\circ} \mathrm{C}$ for 30 seconds, and $72^{\circ} \mathrm{C}$ for 30 seconds for 40 cycles. Transcript abundance was calculated using the delta $\mathrm{Ct}$ method (normalization with $18 S$ ). The primer sequences are listed in Supplemental Table 5.

Histopathology. Specimens of liver, intestine, and skin were provided to 2 pathologists for histopathologic assessment of GVHD. They employed a semiquantitative scoring system for abnormalities known to be associated with GVHD, as described previously (71). Images were obtained with a Leica DM1000 LED (10/0.25 HI PLAN lens, 200× magnification, Leica MC170 HD camera).

Statistics. Survival rates across different groups were compared using the log-rank test. If the data were normally distributed, 2-tailed Student's $t$ test was used for 2-group comparisons, and 1-way ANOVA with Tukey's multiple-comparison test, Bonferroni's multiple-comparison test, or Dunnett's multiple-comparison test was used for multiple comparisons. For data that were not normally distributed, Kruskal-Wallis test with Dunn's multiple-comparison test was used for multiple group comparisons or 2-tailed Mann-Whitney $U$ tests for comparisons of 2 groups. No blinding was done, as objective quantitative assays such as flow cytometry were used. Experimental sample sizes were chosen using power calculations with preliminary experiments and/or were based on previously described variability in similar experiments. Samples that had undergone technical failure during processing were excluded from analyses. Where relevant, recipient mice were randomized before adoptive transfer. $P$ values of 0.05 or less were considered significant.
Study approval. Experimental protocols were approved by Temple University's Institutional Animal Care \& Use Committee (IACUC). Human studies were approved by the Institutional Review Boards of the Children's Hospital of Soochow University, Pennsylvania State University, and the University of Pennsylvania, after obtaining written informed consent.

\section{Author contributions}

YT, LM, SH, and Yi Zhang conceived and designed the project. YT, LM, YW, BL, HY, TB, CA, AL, Yongping Zhang, CZ, JW, SG, SM, EH, DP, Yanyun Zhang, and Yi Zhang performed experiments and analyzed the data. YT, LM, SH, and Yi Zhang designed experiments and analyzed data. Yan Zhou analyzed RNA-seq data. SM, HZ, SG, Yanyun Zhang, YT, LM, AL, SH, EH, DP, and Yi Zhang edited the manuscript. YT, SH, and Yi Zhang wrote and edited the manuscript. The order of co-first authors was determined based on an equal contribution to the study: LM started this project, YT completed this project, and both performed intensive experimental studies and contributed to the writing of this paper.

\section{Acknowledgments}

This study is supported by grants from the NCI (CA172106-01 to Yi Zhang), NHLBI (HL127351-01A1 to Yi Zhang), and NIAID (AI143256-01A1 to Yi Zhang). Clinically relevant studies are supported by grants from the National Science Foundation of China (81770193 and 81970163 to SH).

Address correspondence to: Shaoyan $\mathrm{Hu}$, Department of Hematology, Children Hospital, Soochow University School of Medicine, Suzhou, China. Email: hushaoyan@suda.edu.cn. Or to: Yi Zhang, Fels Institute for Cancer Research and Molecular Biology, Department of Microbiology and Immunology, Lewis Katz School of Medicine, Temple University, Philadelphia, Pennsylvania 19140, USA. Phone: 215.707.8901; Email: yi.zhang@temple.edu.
1. Steinman RM, Banchereau J. Taking dendritic cells into medicine. Nature. 2007;449(7161):419-426.

2. Palucka K, Banchereau J. Dendritic-cell-based therapeutic cancer vaccines. Immunity. 2013;39(1):38-48.

3. Merad M, Sathe P, Helft J, Miller J, Mortha A. The dendritic cell lineage: ontogeny and function of dendritic cells and their subsets in the steady state and the inflamed setting. Annu Rev Immunol. 2013;31:563-604.

4. Reizis B. Plasmacytoid dendritic cells: development, regulation, and function. Immunity. 2019;50(1):37-50.

5. Shortman K, Naik SH. Steady-state and inflammatory dendritic-cell development. Nat Rev Immunol. 2007;7(1):19-30.

6. Conrad C, et al. Plasmacytoid dendritic cells promote immunosuppression in ovarian cancer via ICOS costimulation of Foxp3(+) T-regulatory cells. Cancer Res. 2012;72(20):5240-5249.

7. Labidi-Galy SI, et al. Quantitative and functional alterations of plasmacytoid dendritic cells contribute to immune tolerance in ovarian cancer. Cancer Res. 2011;71(16):5423-5434.

8. Reddy V, Iturraspe JA, Tzolas AC, Meier-Kriesche HU, Schold J, Wingard JR. Low dendritic cell count after allogeneic hematopoietic stem cell transplantation predicts relapse, death, and acute graft-versus-host disease. Blood. 2004;103(11):4330-4335.

9. Fearnley DB, Whyte LF, Carnoutsos SA, Cook $\mathrm{AH}$, Hart DN. Monitoring human blood dendritic cell numbers in normal individuals and in stem cell transplantation. Blood. 1999;93(2):728-736.

10. Vakkila J, Thomson AW, Hovi L, Vettenranta K, Saarinen-Pihkala UM. Circulating dendritic cell subset levels after allogeneic stem cell transplantation in children correlate with time post transplant and severity of acute graftversus-host disease. Bone Marrow Transplant. 2005;35(5):501-507.

11. Mohty M. Impact of plasmacytoid dendritic cells on outcome after reduced-intensity conditioning allogeneic stem cell transplantation. Leukemia. 2005;19(1):1-6.

12. Koyama M, et al. Donor colonic CD103+ dendritic cells determine the severity of acute graft-versushost disease. J Exp Med. 2015;212(8):1303-1321.

13. Leveque-El Mouttie L, et al. Corruption of dendritic cell antigen presentation during acute GVHD leads to regulatory T-cell failure and chronic GVHD. Blood. 2016;128(6):794-804.

14. Banovic T, et al. Graft-versus-host disease prevents the maturation of plasmacytoid dendritic cells. J Immunol. 2009;182(2):912-920.

15. Zeiser R, Blazar BR. Acute graft-versus-host disease - biologic process, prevention, and therapy. NEngl J Med. 2017;377(22):2167-2179.

16. Zhang Y, et al. Tumor necrosis factor (TNF) is a physiologic regulator of hematopoietic progenitor cells: increase of early hematopoietic progenitor cells in TNF receptor p55-deficient mice in vivo and potent inhibition of progenitor cell proliferation by TNF alpha in vitro. Blood. 1995;86(8):2930-2937.

17. Baldridge MT, King KY, Boles NC, Weksberg DC, Goodell MA. Quiescent haematopoietic stem cells are activated by IFN-gamma in response to chronic infection. Nature. 2010;465(7299):793-797.

18. Matatall KA, et al. Chronic infection depletes hematopoietic stem cells through stressinduced terminal differentiation. Cell Rep. 2016;17(10):2584-2595.

19. Ma J, et al. Mammalian target of rapamycin regulates murine and human cell differentiation through STAT3/p63/Jagged/Notch cascade. J Clin Invest. 2010;120(1):103-114.

20. Asselin-Paturel C, Trinchieri G. Production of type I interferons: plasmacytoid dendritic cells and beyond. J Exp Med. 2005;202(4):461-465.

21. Liu C, et al. Plasmacytoid dendritic cells induce 
NK cell-dependent, tumor antigen-specific T cell cross-priming and tumor regression in mice. J Clin Invest. 2008;118(3):1165-1175.

22. Tel J, et al. Natural human plasmacytoid dendritic cells induce antigen-specific T-cell responses in melanoma patients. Cancer Res. 2013;73(3):1063-1075.

23. Devi KS, Anandasabapathy N. The origin of DCs and capacity for immunologic tolerance in central and peripheral tissues. Semin Immunopathol. 2017;39(2):137-152.

24. Audiger C, Rahman MJ, Yun TJ, Tarbell KV, Lesage $\mathrm{S}$. The importance of dendritic cells in maintaining immune tolerance. J Immunol. 2017;198(6):2223-2231.

25. Ganguly D, Haak S, Sisirak V, Reizis B. The role of dendritic cells in autoimmunity. Nat Rev Immunol. 2013;13(8):566-577.

26. Hadeiba H, Sato T, Habtezion A, Oderup C, Pan J, Butcher EC. CCR9 expression defines tolerogenic plasmacytoid dendritic cells able to suppress acute graft-versus-host disease. Nat Immunol. 2008;9(11):1253-1260.

27. Auletta JJ, Devine SM, Waller EK. Plasmacytoid dendritic cells in allogeneic hematopoietic cell transplantation: benefit or burden? Bone Marrow Transplant. 2015;51(3):333-343.

28. Lu Y, et al. IFN-gamma and indoleamine 2,3-dioxygenase signaling between donor dendritic cells and $\mathrm{T}$ cells regulates graft versus host and graft versus leukemia activity. Blood. 2012;119(4):1075-1085.

29. Koyama M, et al. Plasmacytoid dendritic cells prime alloreactive $\mathrm{T}$ cells to mediate graft-versus-host disease as antigen-presenting cells. Blood. 2009;113(9):2088-2095.

30. Waller EK, et al. Improved survival after transplantation of more donor plasmacytoid dendritic or naïve T cells from unrelated-donor marrow grafts: results from BMTCTN 0201. J Clin Oncol. 2014;32(22):2365-2372.

31. Watowich SS, Liu YJ. Mechanisms regulating dendritic cell specification and development. Immunol Rev. 2010;238(1):76-92.

32. Mochizuki K, et al. Programming of donor T cells using allogeneic $\delta$-like ligand 4-positive dendritic cells to reduce GVHD in mice. Blood. 2016;127(25):3270-3280.

33. Rodrigues PF, Alberti-Servera L, Eremin A, Grajales-Reyes GE, Ivanek R, Tussiwand R. Distinct progenitor lineages contribute to the heterogeneity of plasmacytoid dendritic cells. Nat Immunol. 2018;19(7):711-722.

34. Buza-Vidas N, et al. FLT3 expression initiates in fully multipotent mouse hematopoietic progenitor cells. Blood. 2011;118(6):1544-1548.

35. Park IK, et al. Bmi-1 is required for maintenance of adult self-renewing haematopoietic stem cells. Nature. 2003;423(6937):302-305.

36. Collin M, Bigley V. Human dendritic cell subsets: an update. Immunology. 2018;154(1):3-20.

37. Chopin M, et al. Transcription factor PU.1 promotes conventional dendritic cell identity and function via induction of transcriptional regulator DC-SCRIPT. Immunity. 2019;50(1):77-90.e5.

38. Orkin SH, Zon LI. Hematopoiesis: an evolv- ing paradigm for stem cell biology. Cell. 2008;132(4):631-644.

39. Adolfsson J, et al. Upregulation of Flt3 expression within the bone marrow Lin(-)Sca1(+) c-kit(+) stem cell compartment is accompanied by loss of self-renewal capacity. Immunity. 2001;15(4):659-669.

40. Yoshihara H, et al. Thrombopoietin/MPL signaling regulates hematopoietic stem cell quiescence and interaction with the osteoblastic niche. Cell Stem Cell. 2007;1(6):685-697.

41. Thompson BJ, et al. Control of hematopoietic stem cell quiescence by the $\mathrm{E} 3$ ubiquitin ligase Fbw7. JExp Med. 2008;205(6):1395-1408.

42. Shono Y, et al. Bone marrow graft-versus-host disease: early destruction of hematopoietic niche after MHC-mismatched hematopoietic stem cel transplantation. Blood. 2010;115(26):5401-5411.

43. Huang J, et al. Pivotal role for glycogen synthase kinase-3 in hematopoietic stem cell homeostasis in mice. J Clin Invest. 2009;119(12):3519-3529.

44. Sathaliyawala T, et al. Mammalian target of rapamycin controls dendritic cell development downstream of Flt3 ligand signaling. Immunity. 2010;33(4):597-606.

45. Toffalori C, et al. Immune signature drives leukemia escape and relapse after hematopoietic cell transplantation. Nat Med. 2019;25(4):603-611

46. Deng M, et al. LILRB4 signalling in leukaemia cells mediates $\mathrm{T}$ cell suppression and tumour infiltration. Nature. 2018;562(7728):605-609.

47. Thangavelu G, Blazar BR. Achievement of tolerance induction to prevent acute graft-vs.-host disease. Front Immunol. 2019;10:309.

48. Nishimura $\mathrm{R}$, et al. In vivo trafficking and surviva of cytokine-induced killer cells resulting in minimal GVHD with retention of antitumor activity. Blood. 2008;112(6):2563-2574.

49. Anasetti C, et al. Peripheral-blood stem cells versus bone marrow from unrelated donors. $N \mathrm{EnglJ}$ Med. 2012;367(16):1487-1496.

50. Heinrichs J, et al. CD8(+) Tregs promote GVHD prevention and overcome the impaired GVL effect mediated by CD4(+) Tregs in mice. Oncoimmunology. 2016;5(6):e1146842.

51. Reddy P, et al. Histone deacetylase inhibitor suberoylanilide hydroxamic acid reduces acute graft-versus-host disease and preserves graft-versus-leukemia effect. Proc Natl Acad Sci U S A. 2004;101(11):3921-3926.

52. Toubai T, et al. Ikaros deficiency in host hematopoietic cells separates GVL from GVHD after experimental allogeneic hematopoietic cell transplantation. Oncoimmunology. 2015;4(7):e1016699.

53. Hassan M, et al. Flt3L treatment of bone marrow donors increases graft plasmacytoid dendritic cell content and improves allogeneic transplantation outcomes. Biol Blood Marrow Transplant. 2019;25(6):1075-1084.

54. Yu H, Tian Y, Wang Y, Mineishi S, Zhang Y. Dendritic cell regulation of graft-vs.-host disease: immunostimulation and tolerance. Front Immunol. 2019;10:93.

55. Ullrich E, et al. BATF-dependent IL-7R ${ }^{\text {hi }}$ GM$\mathrm{CSF}^{+} \mathrm{T}$ cells control intestinal graft-versus-host disease. J Clin Invest. 2018;128(3):916-930.

56. Tugues S, et al. Graft-versus-host disease, but not graft-versus-leukemia immunity, is mediated by GM-CSF-licensed myeloid cells. Sci Transl Med. 2018;10(469):eaat8410.

57. Piper C, et al. Pathogenic Bhlhe $40^{+}{\mathrm{GM}-\mathrm{CSF}^{+}}^{+}$ $\mathrm{CD}^{+} \mathrm{T}$ cells promote indirect alloantigen presentation in the GI tract during GVHD. Blood. 2020;135(8):568-581.

58. Robb RJ, et al. Type I-IFNs control GVHD and GVL responses after transplantation. Blood. 2011;118(12):3399-3409.

59. Yang YG, Dey BR, Sergio JJ, Pearson DA, Sykes M. Donor-derived interferon gamma is required for inhibition of acute graft-versus-host disease by interleukin 12. JClin Invest. 1998;102(12):2126-2135.

60. Yang YG, Qi J, Wang MG, Sykes M. Donor-derived interferon gamma separates graft-versus-leukemia effects and graft-versus-host disease induced by donor CD8 T cells. Blood. 2002;99(11):4207-4215.

61. Wang H, et al. Paradoxical effects of IFN-gamma in graft-versus-host disease reflect promotion of lymphohematopoietic graft-versus-host reactions and inhibition of epithelial tissue injury. Blood. 2009;113(15):3612-3619.

62. Burman AC, et al. IFNgamma differentially controls the development of idiopathic pneumonia syndrome and GVHD of the gastrointestinal tract. Blood. 2007;110(3):1064-1072.

63. Yi T, et al. Reciprocal differentiation tissue-specific pathogenesis of Th1, Th2, Th17 cells in graft versus host disease. Blood. 2009;114(14):3101-3112.

64. Murphy WJ, et al. Differential effects of the absence of interferon-gamma and IL-4 in acute graft-versus-host disease after allogeneic bone marrow transplantation in mice. J Clin Invest. 1998;102(9):1742-1748.

65. Yang YG, Wang H, Asavaroengchai W, Dey BR Role of interferon-gamma in GVHD and GVL. Cell Mol Immunol. 2005;2(5):323-329.

66. Sathe P, Vremec D, Wu L, Corcoran L, Shortman K. Convergent differentiation: myeloid and lymphoid pathways to murine plasmacytoid dendritic cells. Blood. 2013;121(1):11-19.

67. Zhang Y, et al. Notch signaling is a critical regulator of allogeneic $\mathrm{CD} 4^{+} \mathrm{T}$-cell responses mediating graft-versus-host disease. Blood. 2011;117(1):299-308.

68. He S, et al. Inhibition of histone methylation arrests ongoing graft-versus-host disease in mice by selectively inducing apoptosis of alloreactive effector T cells. Blood. 2012;119(5):1274-1282.

69. Mochizuki K, et al. Delta-like ligand 4 identifies a previously uncharacterized population of inflammatory dendritic cells that plays important roles in eliciting allogeneic $\mathrm{T}$ cell responses in mice. Jimmunol. 2013;190(7):3772-3782.

70. Trapnell C, Hendrickson DG, Sauvageau M, Goff L, Rinn JL, Pachter L. Differential analysis of gene regulation at transcript resolution with RNA-seq. Nat Biotechnol. 2013;31(1):46-53.

71. Cooke KR, et al. Host reactive donor T cells are associated with lung injury after experimental allogeneic bone marrow transplantation. Blood. 1998;92(7):2571-2580. 